\title{
Meson and baryon spectrum for QCD with two light dynamical quarks
}

\author{
Georg P. Engel ${ }^{1}$, C. B. Lang ${ }^{1}$, Markus Limmer ${ }^{1}$, Daniel Mohler ${ }^{1,2}$, and Andreas Schäfer ${ }^{3}$ \\ (BGR [Bern-Graz-Regensburg] Collaboration) \\ ${ }^{1}$ Institut für Physik, FB Theoretische Physik, Universität Graz, A-8010 Graz, Austria \\ ${ }^{2}$ TRIUMF, 4004 Wesbrook Mall Vancouver, BC V6T 2A3, Canada \\ ${ }^{3}$ Institut für Theoretische Physik, Universität Regensburg, D-93040 Regensburg, Germany
}

(Dated: November 4, 2018)

\begin{abstract}
We present results of meson and baryon spectroscopy using the Chirally Improved Dirac operator on lattices of size $16^{3} \times 32$ with two mass-degenerate light sea quarks. Three ensembles with pion masses of 322(5), 470(4) and 525(7) MeV and lattice spacings close to $0.15 \mathrm{fm}$ are investigated. Results for ground and excited states for several channels are given, including spin two mesons and hadrons with strange valence quarks. The analysis of the states is done with the variational method, including two kinds of Gaussian sources and derivative sources. We obtain several ground states fairly precisely and find radial excitations in various channels. Excited baryon results seem to suffer from finite size effects, in particular at small pion masses. We discuss the possible appearance of scattering states, considering masses and eigenvectors. Partially quenched results in the scalar channel suggest the presence of a 2-particle state, however, in most channels we cannot identify them. Where available, we compare our results to results of quenched simulations using the same action.

PACS numbers: 11.15.Ha, 12.38.Gc
\end{abstract}

\section{INTRODUCTION}

The overwhelming majority of hadronic states in the Particle Data Group's collection are hadron excitations [1]. QCD as the theory of strong interactions should give the whole spectrum of hadrons, based on the few quark mass parameters and a scale. So far, the lattice regularized form of QCD provides the only known way to perform ab-initio calculations of these observables. Only recently there have been lattice calculations with dynamical quark masses close to their physical values; most calculations still rely on extrapolations from unphysically heavy quarks. A reliable determination of the excited states still remains a hard challenge. The present calculation is another step in this enterprise.

For decades a lattice realization of chiral symmetry has been an obstacle; quite early it was observed [2] that a solution might be Dirac operators obeying an non-linear relation, the so-called Ginsparg-Wilson (GW) condition, overlooked for many years. Meanwhile we know several Dirac operators obeying that condition. One of them, the overlap operator [3, 4], has an explicit construction based on a domain-wall approach [5, [6] in the limit of infinite extent of a fifth dimension. Another formulation (perfect fermions) is formally exact [7], but, like other approaches, can be constructed only in an approximate version [8]. The lattice version of chiral symmetry underlying the GW relation has been given in [9]. GWDirac operators are numerically expensive to construct but have nice properties like protection from additive mass renormalization or automatic $\mathcal{O}(a)$ improvement.

Due to the construction method, which, e.g., in the case of overlap fermion, involves computation of an operator sign function, simulations with dynamical GW- fermions are very expensive, typically two orders of magnitude more expensive than simulations with the simple, improved Wilson Dirac operator. A problem especially apparent for GW-fermions concerns tunneling between different topological sectors. Due to the Atiyah-Singer theorem we know that topological sectors of the gauge configurations are related to the net number of exact zero modes of the Dirac operator (counted according to their chirality signature).

The so-called Chirally Improved (CI) Dirac operator is an approximate solution of the Ginsparg-Wilson equation [10, 11]. Its construction is based on a formal parameterization of the Dirac operator inserted in the GWequation and solved in truncated from. This fermion action has already been used extensively in simulations by the BGR-collaboration in the quenched approximation. It was found that at least in quenched simulations the $\mathcal{O}\left(a^{2}\right)$ corrections for baryon masses are small [12] and that renormalization constants behave similar to an exact chirally symmetric action [13].

In this paper we present results of dynamical simulations with two mass-degenerate light quarks using this action. The parametrization, as well as details of the simulation and some early results are given in [14, 15. The small discretization errors allow us to use rather coarse lattices in order to save computational costs.

Here we discuss the results concerning several meson and baryon masses derived on the gauge field configurations of [15]. We present results of ground states as well as excited states, making use of the variational method [16, 17]. In addition to the light (dynamical and valence) quarks we also consider another, heavier valence (strange) quark and include the strange mesons and baryons in our analysis. With the presently available data we neither perform a continuum limit nor an 
infinite volume limit. This may be justified considering the small discretization errors and that our values for $m_{\pi} L$ should be large enough to expect small finite volume effects due to the pion cloud. However, in addition to pions "wrapping around the universe", finite volume effects can appear when considering large hadrons which may not "fit in the box". A possible interpretation of our results involves such squeezing effects in case of the excited baryons. Motivated empirically, extrapolation to the physical pion mass is performed naively, with a fit linear in the pion mass squared. Preliminary results have been presented in [18].

Recent results on light and strange hadron spectroscopy with focus on excited states following different approaches can be found in [19 33].

This paper focuses on results for hadron masses from dynamical CI-simulation and is organized as follows: In Sect. II we review the setup and parameters of the simulation, followed by the discussion of the methods to extract hadron masses in Sect. [II] Extrapolation to physical quark masses and sources for systematic errors are discussed in Sect. IV] In Sections V] and VI we then present our results on hadron masses. We conclude in Sect. VII.

\section{SIMULATION DETAILS}

\section{A. Fermion action and gauge action}

All details of the simulation method and our checks for equilibration and autocorrelation are given in [15]. For completeness we summarize the essential features here.

We use the Chirally Improved Dirac $\left(D_{\mathrm{CI}}\right)$ operator [10, 11]; this is an approximate solution to the GinspargWilson equation. It is obtained by insertion of the most general ansatz for a Dirac operator into the GW equation and comparison of the coefficients. This leads to an algebraic set of coupled equations, which can be solved numerically. The accuracy of the approximation depends on the number of terms included after the truncation, in our case considering paths to neighbors up to a maximum path length of 4 lattice units. The paths and coefficients used are found in the appendix of [15]. In principle, one could optimize the coefficients in the parametrization for each gauge coupling and quark mass value with respect to chiral symmetry. However, defining the setup this way, the predictive power of the simulation is weakened, and, furthermore, comparison of different sets of gauge ensembles is more complicated. We therefore decided to use the same paths and coefficients in all our dynamical runs and thus the bare Dirac operator is the same in all discussed ensembles. This leads to additive mass renormalization which is corrected for by determining the PCAC (partially conserved axial current) mass, also called AWI (axial Ward identity) mass for each ensemble.

We include one level of stout smearing [34] as part of the definition of $D_{\mathrm{CI}}$ in order to improve the fermion action further. The parameters of stout smearing are adjusted such that the value of the plaquette is maximized ( $\rho=0.165$ in the notation of Ref. [34]).

It was found that the combination of the $D_{\mathrm{CI}}$ with the tadpole-improved Lüscher-Weisz gauge action shows nice properties [11]. We use this gauge action as discussed in [15].

\section{B. Algorithm}

We generate the dynamical configurations with a Hybrid Monte-Carlo (HMC) algorithm [35], with the implementation for $D_{\mathrm{CI}}$ described in [14]. Performance improvement is obtained by Hasenbusch mass preconditioning [36] with two pseudofermions. Further improvement is achieved by the use of a chronological inverter by minimal residue extrapolation [37] and a mixed precision inverter [38]. A discussion of the autocorrelation time, the eigenvalues of the Dirac operator and the topological sector of the generated configurations is found in 15]. We choose to analyze every fifth configuration and neglect any remaining weak correlations. The distribution of the eigenvalues of $D_{\mathrm{CI}}$ indicates that we may simulate small pion masses on relatively coarse lattices. The algorithm was found to show frequent tunneling through topological sectors.

The gauge configurations are determined on an SGI ALTIX-4700 (a machine with a peak rate of $62.3 \mathrm{TFlop} / \mathrm{s}$ for 9728 processors) using for each configuration (i.e., one unit of HMC-time) a total amount (summed over the processors used in parallel) of $\mathcal{O}(60)$ CPUh for set A and up to $\mathcal{O}(120)$ CPUh for set $\mathrm{C}$. The quark propagators for the analysis are computed with a multi-mass solver and thus the CPU time depends only on the smallest (the dynamical) quark mass. Computing the quark propagators for one configuration and 60 sources (five source types, cf., Sect. ПIВ and 12 Dirac-color sources each) takes approximately the same amount of CPU time as for generating one gauge configuration. A recent comparison for different actions can be found in Ref. [39].

\section{Simulation parameters}

We use lattices of size $16^{3} \times 32$ at three different values of the gauge coupling $\beta_{L W}$ and bare quark mass parameter $m_{0}$, see Table【. The lattice spacing is determined via the static quark potential, using a Sommer parameter of $r_{0, \exp }=0.48 \mathrm{fm}$. The bare simulation parameters are chosen such that the lattice spacing is of approximately the same magnitude in all three ensembles. Hence their physical volume is of the same size as well $(\approx 2.4 \mathrm{fm})$.

Considering the chiral extrapolation, many calculations make use of the so-called mass independent scheme (cf., the discussion in [40]). In this scheme the lattice spacing is determined (for fixed bare gauge coupling) in the chiral limit and assigned to all ensembles with that 


\begin{tabular}{cccccccc}
\hline \hline set & $\beta_{L W}$ & $m_{0}$ & configs & $a[\mathrm{fm}]$ & $m_{\pi}[\mathrm{MeV}]$ & $m_{A W I}[\mathrm{MeV}]$ & $m_{\pi} L$ \\
\hline A & 4.70 & -0.050 & 100 & $0.151(2)$ & $525(7)$ & $43.0(4)$ & 6.4 \\
B & 4.65 & -0.060 & 200 & $0.150(1)$ & $470(4)$ & $35.1(2)$ & 5.7 \\
C & 4.58 & -0.077 & 200 & $0.144(1)$ & $322(5)$ & $15.0(4)$ & 3.7 \\
\hline \hline
\end{tabular}

TABLE I: Bare parameters of the simulation: Three ensembles (A,B,C), at different gauge coupling $\beta_{L W}$ and quark mass parameter $m_{0}$. The number of configurations, lattice spacing from the static potential assuming a Sommer parameter of 0.48 $\mathrm{fm}$, the pion mass, the (non-renormalized) AWI-mass and the dimensionless product of the pion mass with the physical lattice size are given. For more details see $[15]$.

gauge coupling. Since so far we only have one mass value at each gauge coupling, we make use of a mass dependent scheme, differing by $\mathcal{O}(a)$ corrections.

\section{METHODS IN HADRON SPECTROSCOPY}

\section{A. Variational method}

Excited state contributions are suppressed by $\mathcal{O}\left(\mathrm{e}^{-\Delta E}\right)$ in the hadron correlators. However, the so-called variational method [16, 17] allows to extract excited states in principle. This method has been used extensively by the BGR-collaboration [15, 41 45] and has gained popularity in recent years.

A simple hadron interpolating field operator with the right quantum numbers will have a correlation function that asymptotically decays with $e^{-E t}$ where $E$ denotes the ground state energy. However, at finite time distance there will be contributions from excited states embedded in the continuum of scattering states. On a lattice with finite spatial extent the corresponding energy spectrum is discrete. In case there are no dynamical quarks, the eigenstates may be interpreted as bound states of the valence quarks in the interpolator. In the fully dynamical situation such a simple interpretation is not possible, since mixing with all many particle states with the same quantum numbers can occur. The eigenenergy levels are related to the scattering phase shifts of the coupling channels [46-49] and the space of lattice hadron interpolators has to be large enough, in order to represent the possible intermediate states with sufficient quality.

Given a set of $N$ interpolators (with given quantum numbers) in the variational method one constructs the corresponding correlation matrix

$$
\begin{aligned}
C_{i j}(t) & =\left\langle 0\left|O_{i}(t) O_{j}^{\dagger}\right| 0\right\rangle, \\
& =\sum_{k=1}^{N}\left\langle 0\left|O_{i}\right| k\right\rangle\left\langle k\left|O_{j}^{\dagger}\right| 0\right\rangle \mathrm{e}^{-t E_{k}} .
\end{aligned}
$$

The idea is to offer a basis of convenient interpolators, wherefrom the system chooses the linear combinations closest to the physical eigenstates. Diagonalization of the correlation matrix of the interpolators thus allows to disentangle the physical states, if the state of interpola- tors is sufficiently complete. The generalized eigenvalue equation

$$
\begin{aligned}
C(t) \vec{v}_{k} & =\lambda_{k}\left(t, t_{0}\right) C\left(t_{0}\right) \vec{v}_{k} \\
\lambda_{k}\left(t, t_{0}\right) & \propto \mathrm{e}^{-\left(t-t_{0}\right) E_{k}}\left(1+\mathcal{O}\left(\mathrm{e}^{-\left(t-t_{0}\right) \Delta E_{k}}\right)\right)
\end{aligned}
$$

gives the energies of the eigenstates, where $\Delta E_{k}$ is the distance of $E_{k}$ to the closest state. In the interval $t_{0} \leq$ $t \leq 2 t_{0}$ it would be determined by the distance to the first neglected state $E_{N+1}$ [50]. However, we use small values for $t_{0}$ ( 1 or 2 ), since otherwise the quality of the diagonalization decreases. We therefore determine the eigenvalues in a larger window of $t$-values.

The corresponding eigenvectors represent the linear combinations of the given interpolators which are closest to the considered physical states at each time slice. Hence they may be used to derive some information on the composition of the physical modes [51, 52.

Obviously, the number of interpolators should be large enough, they should be independent and have overlap primarily with the low modes of the theory, in order to reduce contamination from highly excited states. In actual calculations, including more interpolators unfortunately increases the statistical noise in the diagonalization. Thus, the optimal choice is usually to use only those interpolators, which show good overlap with the low physical modes.

\section{B. Quark source smearing}

Hadron correlation functions are built from quark propagators $D^{-1}$, which are computed by inverting the Dirac operator on a given quark source. Extended sources improve the signal and also allow for a larger operator basis in the variational method. We use three different kinds of sources: narrow $(0.27 \mathrm{fm})$, wide $(0.55 \mathrm{fm})$ and a ( $P$ wave like) derivative source.

The sources are computed using Jacobi smearing [53, 54]: A point-like source is smeared out by applying a 
polynomial of the hopping term,

$$
\begin{aligned}
S_{\kappa, K}= & \sum_{n=0}^{K} \kappa^{n} H^{n} S_{0}, \\
H(\vec{n}, \vec{m})= & \sum_{j=1}^{3}\left(U_{j}(\vec{n}, 0) \delta(\vec{n}+\hat{j}, \vec{m})\right. \\
& \left.\quad+U_{j}(\vec{n}-\hat{j}, 0)^{\dagger} \delta(\vec{n}-\hat{j}, \vec{m})\right),
\end{aligned}
$$

where $S_{0}$ denotes the point source. The resulting source shape is approximately Gaussian. The parameters $\kappa$ and $K$ are tuned (for each ensemble of configurations) such as to ensure approximately the same source width in all ensembles. Narrow (wide) sources will be denoted by quark subscripts $n(w)$ in the remainder of this paper.

The derivative sources, $S_{\partial_{i}}$, are obtained by applying the covariant difference operators on the wide source, $S_{w}$ [45],

$$
\begin{aligned}
P_{i}(\vec{x}, \vec{y}) & =U_{i}(\vec{x}, 0) \delta(\vec{x}+\hat{i}, \vec{y})-U_{i}(\vec{x}-\hat{i}, 0)^{\dagger} \delta(\vec{x}-\hat{i}, \vec{y}), \\
S_{\partial_{i}} & =P_{i} S_{w},
\end{aligned}
$$

where $\hat{i}$ is one of the spatial directions. The derivative sources were found to be crucial for some states [55], as will be confirmed in this paper as well. In the following, derivative sources are indicated by the subscript $\partial_{i}$ of the quark field.

\section{Constructing the interpolators}

As already mentioned, we construct several interpolators in each channel in order to be able to extract excited states using the variational method. All sources are located in a single time slice and built on configurations which have been HYP-smeared in the spatial directions three times [56]. The main motivation for link smearing is suppression of UV-fluctuations which manifest themselves, e.g., in the distribution of the plaquette. Thus, the parameters of the spatial HYP-smearing have been optimized by a trade-off between a maximum average plaquette and a maximum of the minimum plaquette, partly following the arguments of [57, 58]. We obtained the parameters $\alpha_{1}=0.8$ and $\alpha_{2}=0.4$, where $\alpha_{1}$ is the parameter in the last step of the smearing algorithm, where the center link is smeared.

The center positions of the quark sources are shifted for subsequent configurations in order to decrease statistical correlation of the data. The interpolators at the sink are projected to zero momentum, thus for sufficiently many configurations the sum projects to propagators of zero momentum hadrons due to translation invariance. Tables of the interpolators are found in Appendix A

There exists another approach for interpolator construction developed recently, called "distillation" 59], which we do not follow here. Recent results on hadron spectroscopy following this approach are found in 30, 32.

\section{Meson interpolators}

We consider isovector-mesons, thus there are no disconnected diagrams. Using spatially isotropic sources ( $n$ and $w$ ), the quantum numbers of an interpolator are determined by the combinations of the spinor components (Dirac content). This restricts the meson to just a few (non-exotic) channels of spin $\leq 1$. A way to enlarge the basis of interpolators and access higher spin states is given by considering the direct group product of spinor and spatial structure. The decomposition to the irreducible representations then leads to interpolators with definite quantum numbers 60 64. We realize a nontrivial spatial structure by using the derivative sources, which transform according to the lattice spin irreducible representation $T_{1}$.

Depending on the quark content and the implementation of the derivative sources, symmetrization of the interpolators is needed in order to have definite $C$-parity. Hence, light meson interpolators are symmetrized properly, while symmetrization is omitted in the strange meson sector (see Appendix A). Our strange meson correlator calculation omits cross correlation matrix elements corresponding to interpolators with different $C$ parity quantum numbers in the limit of degenerate quark masses. Therefore, when analyzing strange mesons, we have to restrict ourselves to subsets of interpolators sharing the same $J^{P C}$ quantum numbers in the limit of degenerate quark masses.

\section{Baryon interpolators}

For the construction of baryon interpolators we use only Gaussian smeared quark sources $(n, w)$. In case of the nucleon, $\Sigma$ and $\Xi$ we use three different Dirac structures, in case of the $\Delta$ and $\Omega$ only one. Since a baryon is built from three valence quarks, there are $2^{3}=8$ possible smearing combinations. If there is isospin symmetry, some of the resulting 8 interpolators are very similar to others, which we thus prune from the considered set of interpolators. We end up with 18 interpolators in the nucleon channel, 6 in the $\Delta$ and $\Omega$ channels and 24 in the $\Sigma$ and $\Xi$ channels (see Appendix $\mathrm{A}$ ). We project to definite parity in each channel.

\section{Energy levels}

In full QCD calculations the single hadron states couple to channels with two or more hadrons, like the even number of pions in the $\rho$ sector. Although the original hadron is projected to its rest frame, the scattering states have internal relative momenta. For finite spatial extension the admissible values of the momenta depend on the spatial size and the (Euclidean) discrete energy levels are related to the phase shift of the scattering states. In the elastic region this relationship has been discussed in $46-$ 
[49].

Neglecting further interactions of the hadronic bound states, the energy level $E(A, B)$ for two free hadrons reads

$$
\begin{aligned}
& E(A(\vec{p}), B(-\vec{p}))= \\
& \left(\sqrt{m_{A}^{2}+|\vec{p}|^{2}}+\sqrt{m_{B}^{2}+|\vec{p}|^{2}}\right)(1+\mathcal{O}(a p)) .
\end{aligned}
$$

The hadrons $A$ and $B$ have back-to-back momenta since the whole state is projected to zero momentum. In the infinite volume limit, there is a continuum of scattering states. In a finite box, the momentum $\vec{p}$ can take only discrete values, determined by the boundary conditions, $a \vec{p}=2 \pi\left(n_{x}, n_{y}, n_{z}\right) / L$, where $L=16$ in the present work. In the $S$ wave, the lowest 2-particle state level thus shows vanishing relative momentum, while in the $P$ wave, the lowest 2-particle state level has a momentum of $a|\vec{p}|=$ $2 \pi / L$.

The lowest energy levels of the fictitious two-freehadron-state for each ensemble are indicated in the figures using symbols $\times$ and + , provided the energy levels are in the range of our investigation and can be estimated from our results. The corresponding non-correlated statistical uncertainty, neglecting the hadronic interaction and finite volume effects, is of the magnitude of 5 to 60 $\mathrm{MeV}$. For clarity, these error bars are suppressed in the figures if the error is smaller than $30 \mathrm{MeV}$, and furthermore, we always omit the error bar in case of continuous curves of many-particle states in the figures.

In many of our hadron correlators, due to the parameters of the simulation and the resulting pion masses, twoparticle intermediate states will have an energy larger than the ground state energy. As an example, the $\rho$ could formally couple to two pions with relative momentum (to build the $P$ wave) but this is forbidden for kinematical reason in our case, even for the lightest pion mass of ensemble C. There may be a slight shift of the lowest energy level due to avoided level crossings, though. However, the higher levels could well be due to two-particle states. In the $\rho$ sector one would expect such an energy level between the ground state and a possible $\rho^{\prime}$. In most other cases the expected two-particle state levels are at least close to other observed possible resonance state levels. Except for partially quenched results in the light scalar channel, we do not observe such scattering state levels, or, if they are there, we cannot distinguish them from single particle states.

A possibility to shed some light on the nature of the state is to monitor the eigenvectors $\vec{v}_{k}$ of Eq. 5 of the state when varying parameters of the simulation. Ideally, one compares the eigenvectors for several dynamical simulations, but also partially quenched data can yield some information. Since effects from partial quenching can shift the energy level, corresponding results may also allow to extract further information about the state.

For example, in the light and strange scalar channels we find that our partially quenched results are well described by partially quenched formulae of 2-particle states 65. However, at the dynamical point our data do not allow for a unique interpretation. Both, the resonance and the scattering state may be present and contribute to the measured energy level.

In the case of the negative parity baryon channels, positive parity baryons and pseudoscalars might form scattering states whose energy levels are consistent with our results. While the extracted masses slightly favor the scattering states, the eigenvectors do not allow for an interpretation in terms of a level crossing and thus do not confirm the picture of a scattering state at small pion masses, either.

An explanation for missing scattering states would be weak coupling to the interpolators considered. In case of the $S$ wave, there is a noteworthy amplitude already at small momenta, while in case of the $P$ wave higher momenta are needed. This may explain why we see possibly a 2-particle signal in the $S$ wave of $a_{0}\left(J^{P C}=0^{++}\right)$, but not, e.g., an additional energy level between the ground state and $\rho^{\prime}$ in the $P$ wave of $\rho\left(1^{--}\right)$. Consideration of explicit two-hadron interpolators may help.

It is known that channels with two or more particles are suppressed by factors $\mathcal{O}\left(1 / L^{3}\right)$ 66, 67]. This suppression comes on top of the generic suppression of the excited states. Even in kinematical situations where already the ground state couples to scattering states, it turned out, that one has to include both, one- and two-particle states, in the set of interpolators in order to see clear signals 68]. For such attempts, see 69 73]. Unfortunately, the computation of cross-correlations of one- and two-particle states intrinsically includes disconnected diagrams, which are technically demanding and thus not considered here.

\section{COMPARING TO EXPERIMENT}

\section{A. Chiral Extrapolation}

We simulate at pion masses larger than the physical one. Therefore we have to rely on some extrapolation towards small quark masses, if we want to make predictions at the physical point. The analytic form of the chiral extrapolation depends on the path taken in the parameter space of, e.g., the lattice spacing, the quark mass, the gauge coupling and the volume. In chiral perturbation theory (ChPT) [74, 75] the quark mass is the only varying parameter. The lattice spacing and the volume are assumed to remain constant. In the mass-independent scheme (cf., the discussions in [40, 76]) the scale (the physical value of the lattice constant) is set by extrapolating the lattice spacing towards the chiral limit along some path in parameter space (e.g., constant bare gauge coupling). The extrapolated value is then used for all ensembles along that path.

Since we have only one ensemble at each value of the coupling, we cannot use this mass-independent scheme. Hence, our path of extrapolation in parameter space implies a mass-dependent scheme and formulae of ChPT 
would have to be adjusted. Nevertheless, we assume our path to be close to the one in the mass-independent scheme, and expect that the analytic form of the chiral extrapolation should be similar, although with different expansion coefficients. Therefore, we perform chiral fits linear in the pion mass squared for all results and discuss possible other fit forms in certain cases. Note that the fits include only the three dynamical points, the partially quenched points are left out. In the figures, the solid black curve shows the chiral extrapolation, the dashed lines delimit the region of one standard deviation. The results of the chiral fits are summarized in Fig. 29.

\section{B. Systematic Effects}

We set the scale via the static potential and the Sommer parameter (Sect. 【C) with $r_{0}=0.48 \mathrm{fm}$. In the literature also other values have been used. We have only one dynamical quark mass for each value of the gauge coupling and thus cannot extrapolate to the chiral limit in order to define the scale in the mass independent scheme. More dynamical points would be desirable for a more reliable chiral extrapolation and will be included in future calculations.

The strange quark is considered as a valence quark only. In view of results including full strange quark dynamics (e.g., 77]) we find, at least for the ground states, no noticeable difference in the mass range considered here.

Discretization effects have been discussed for baryons in the quenched simulation, where, due to the improvement of the used action, only small $\mathcal{O}\left(a^{2}\right)$ corrections have been identified [12]. In oder to confirm this for the dynamical simulation we would have to perform our study at several lattice spacings and lattice volumes. This is a future task. In the extrapolation to the physical quark masses, using the mass-dependent scheme, we disregard discretization errors and discuss finite volume effects only qualitatively.

The physical size of our spatial lattice volume is $\approx$ $2.4 \mathrm{fm}$. Finite volume effects due to "pions wrapping around the world" are expected to be small in ensemble $\mathrm{A}$ and $\mathrm{B}\left(m_{\pi} L>4\right)$, however, they could be significant in ensemble $\mathrm{C}\left(m_{\pi} L \approx 3.7\right)$. Squeezing of large hadrons, which do not "fit in the box", may be an obstacle in the case of excited baryons. Indeed, we seem to observe such effects. Studies with a larger volume are planned.

A subtle issue is the choice of interpolators for the various hadron states. For sufficiently large and complete sets and exhaustive statistics the variational method would produce the eigenmodes sufficiently well. In our case we are restricted to few interpolators and modest statistics. We attempt to optimize this situation by choosing suitable subsets of interpolators, as discussed, motivated by plateaus in the eigenvector components and exponential decay of the eigenvalues in some fit range. This brings in certain systematic effects, which will be reduced only by enhancing both, statistics and the set of interpolators. We discuss our choice for all hadrons considered subsequently. Indeed, in some cases we find sizeable dependence on the chosen sets.

Another possible systematic influence comes from choosing $t_{0}$ in the variational method and the fit range for the generalized eigenvalues. In principle, that impact can be estimated by choosing several values of $t_{0}$ and varying the fit range. For the final fit one should then choose a window where this impact is negligible. However, in practice the corresponding choices are restricted by the given signal-to-noise ratio for coarse lattices and weak signals. We use $t_{0}=1$ in most (and $t_{0}=2$ in a few) cases and perform a fit in the maximum range possible, which is, however, often limited to 3 or 4 points for noisy observables.

\section{RESULTS: LIGHT HADRONS}

Following the idea of the variational method, a large basis of interpolators should be optimal. In practice, one finds that increasing the number of interpolators also implies an increased statistical noise. One thus has to find an optimal balance when choosing the basis for the variational method.

In each channel we take the subset of interpolators, which yields the optimum plateaus of effective masses. In order to find this subset, we first look at the diagonal elements of the correlation matrix, which represent the autocorrelation of the interpolators used. From that data one has a first hint which interpolators are candidates for such an optimal subset. To be more concrete, we extract information about the interpolators such as to which physical state they couple dominantly, how strongly they are affected by contamination of excited states and until which time separation the signal of the corresponding physical state is reliable.

Usually, the so obtained set of interpolators includes a number of interpolators which are known to have rather large overlap with each-another, due to, e.g., their common Dirac structure. This in turn means that they are far from being orthogonal and thus not well-suited to build a basis of the variational method. Thus, we proceed by singling out subsets, applying the criterion of maximum orthogonality, i.e., least overlap. Doing so, we obtain a number of candidate subsets, all of which are analyzed with the variational method. The fit range of the plateau is chosen from the first point without contaminations from higher states until the plateau breaks down or the noise gets too large. This qualitative criterion is made more precise by choosing an optimal $\chi^{2}$ as quantitative criterion for the fit.

The actual values of the energy levels are then determined by an overall exponential, correlated fit to the eigenvalues at all time slices in the fit range.

This range is validated by comparison with the corresponding plateau of the eigenvectors. The variational 
method simultaneously yields results for several states using one set of interpolators. However, we find that in many cases the signal can be improved by considering different sets for different states. The extracted masses of such different sets agree well within error bars, but the noise is reduced. Theoretically, one would expect to find improvement by joining both sets of interpolators, but in practice this means an increase of the noise, which in most cases is the stronger effect. We show a plot of the effective mass of the $\rho$ meson as one example for consistent results for two sets (see Fig. 4).

We present results on three ensembles of gauge configurations (cf., Table I). In all plots, filled symbols denote dynamical results and open symbols denote partially quenched results, where the valence quark mass is always larger than the sea quark mass. The symbols $\times$ and + represent energy levels of free scattering states, neglecting hadronic interaction and finite volume effects. The scale of the vertical axis is set by the lattice spacing, as discussed, unless stated otherwise. The scale of the abscissa is set using the results for the pion mass squared. The tables of the corresponding interpolators are found in the appendix. The chosen subset of interpolators is stated in the caption of the figures.

\section{A. Mesons with light quarks}

We simulate two mass-degenerate light quarks and thus use the symmetrized meson interpolators found in Appendix A.

\section{The $0^{-+}$channel: $\pi$}

In Fig. 1 the mass of the pion ground state is shown as function of $M_{\pi}^{2}$ to indicate the statistical errors. In the variational method, the observation range of the excited states in this channel is limited by the backwards-running pion (see also the discussion in [15]). This leads to short plateaus for small pion masses and weakens the signal in our simulations. Nevertheless, we find a clear signal of the first excited state, compatible with experimental data. The signal weakens towards smaller quark masses, thus the fit at the dynamical point is motivated by the plateau at partially quenched points and the behavior of the eigenvalues (see Fig. 2). We find no indication of an intermediate three pion state signal; a $\pi \rho$ signal would be expected at even higher energy values. The excited state signal is expected to improve when using a lattice with a larger time extent, in particular in the case of ensemble C. We do not see a reliable and stable signal of the second excitation. The quenched results using the same action [45] showed, within the errors, similar behavior as set A.

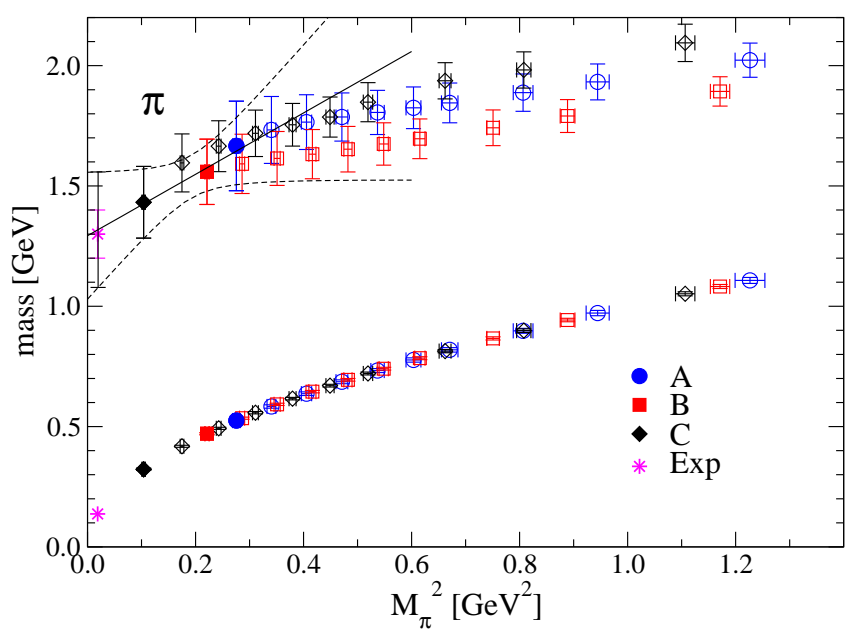

FIG. 1: Mass plot for the $0^{-+}$channel (pion), ground state and first excitation. Filled symbols denote dynamical results, open symbols denote partially quenched results. Parameters of the three ensembles A, B and C are found in Table I The list of the interpolators is found in the appendix. Results for the pion ground state are used to set the scale on the abscissa, here and in other figures as well. The ground state is measured using interpolator (1), the excitation using interpolators $(3,5,9,10)$ in ensemble A, $(1,6,9,10)$ in B and $(3,7,8,11)$ in $\mathrm{C}$.

\section{The $1^{--}$channel: $\rho$}

We find an excellent plateau for the ground state and an excited state signal compatible with experimental data (see Fig. 3). Here, the excitation signal improved using $t_{0}=2$, which may indicate more contamination from excited states in this particular channel. We decided to extract the ground state result from the set of interpolators $(1,4)$, which displays a better plateau than the combination used for the excited state. The consistency of the ground state from $(1,4)$ and the sets chosen for the excitation is shown in Fig. 4. In ensemble B we furthermore see a signal of the second excitation compatible with the $\rho(1700)$. The physical $\rho$ meson is a resonance which can decay into two pions with relative momentum. The energy of the corresponding lowest scattering state is determined by the mass of the pion and the minimum non-vanishing momentum, defined by the finite spatial extent of the lattice. On the lattice, for our ensemble parameters the energy of this scattering state would be above the mass of the $\rho$ meson. Hence the ground state of the channel is dominated by the $\rho$ meson, which does not decay and is therefore called "stabilized". One might expect, however, a two-pion intermediate state between the $\rho$ ground state energy and the signal associated to $\rho^{\prime}$, but no such state is observed here. It is suggested that the coupling of the used interpolators to two-particle states is strongly suppressed in the $P$ wave.

In principle, $\mathrm{T}_{1}$ interpolators may couple to spin 3 states with energy levels close to $\rho^{\prime}$. However, from the 

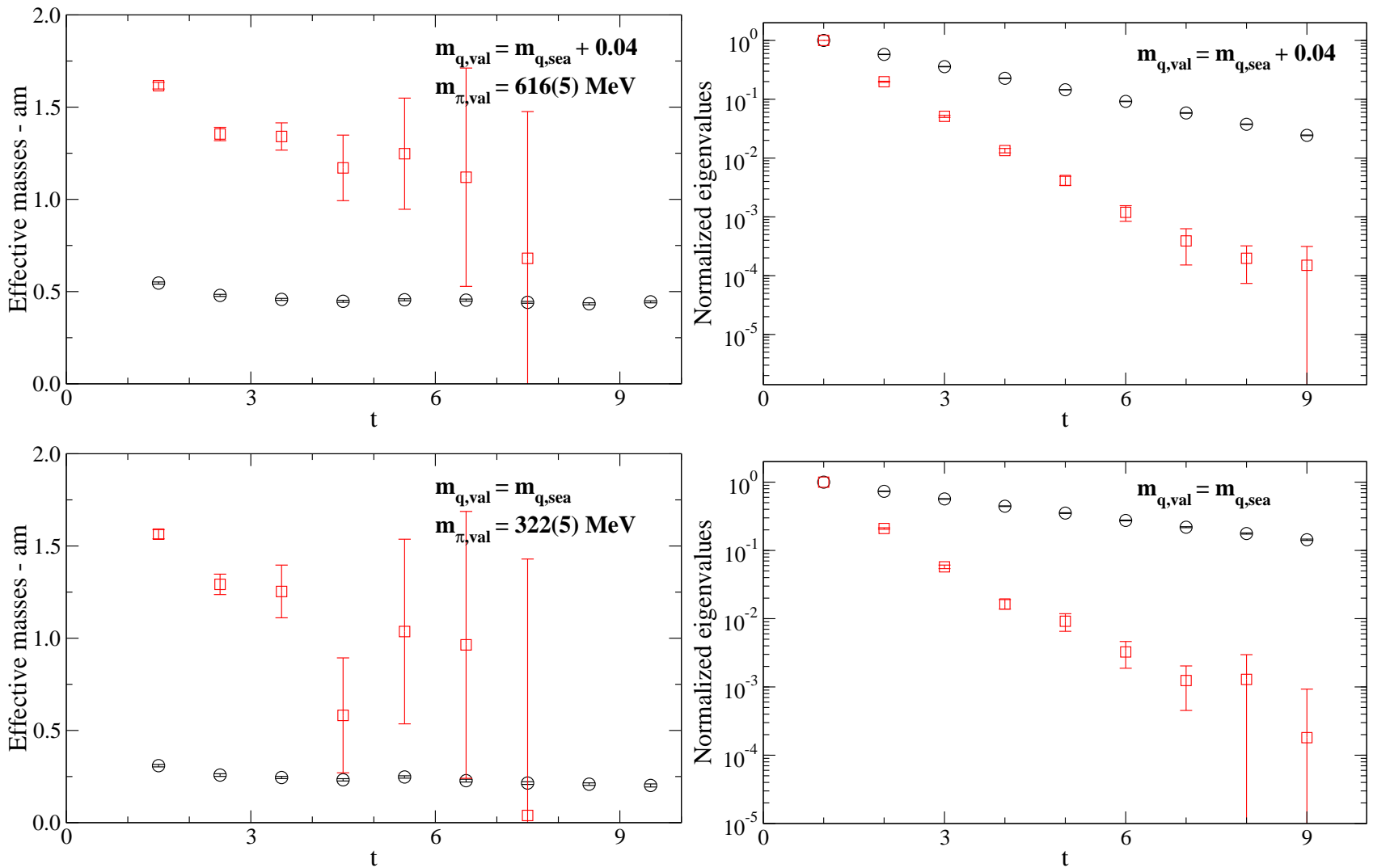

FIG. 2: Effective mass plots (lhs) and normalized eigenvalues (rhs, logarithmic scale) for the $0^{-+}$channel (pion) at the dynamical point (bottom) and at a partially quenched point (top) of ensemble C, using interpolators $(3,7,8,11)$. Ground state and the first excitation data are shown. The corresponding valence quark mass parameter and the fictitious pion mass are indicated in the figure. The eigenvalues suggest that the first excitation can be fitted from $t=3$ to $t=7$, at $t=8$ we find a kink at partially quenched data and even a loss of the signal at the dynamical point. The effective masses are proportional to the derivative of the logarithm of the eigenvalues, thus showing huge error bars and very bad plateaus in case the eigenvalues are tumbling. The fit to the excitation at the dynamical point is motivated by the plateau at partially quenched points and the almost stable behavior of the eigenvalues. We remark that the final fit is an overall exponential fit of the eigenvalues for all points in the chosen range $3 \leq t \leq 7$.

naive continuum limit of our interpolators we expect such coupling to be small.

Using only "gaussian-type" interpolators (i.e., without derivative sources), the energy levels of the first excitation are found to deviate slightly from the results presented here. A possible reason could be mixing with the nearby higher excited states, or an early breakdown of the plateau which can complicate the identification of a correct fit range. Moving the used fit range 3-6 to $2-5$ the excitation level increases.

Comparing with quenched results using the same action [45], we find that the dynamical $\rho$ ground state comes out significantly lighter than its quenched counterpart, which, however, is partially due to the different Sommer parameter value used in the quenched analysis $\left(r_{0}=0.5 \mathrm{fm}\right)$. Again, the first excitation of the quenched simulation is compatible with set $\mathrm{A}$ of the dynamical case. The dynamical points $\mathrm{B}$ and $\mathrm{C}$ indicate a steeper slope pointing towards the experimental results.

The isospin singlet vector meson $\phi$ has mainly $s \bar{s}$ content and disconnected parts are suppressed ("Zweigforbidden", cf., the decay channels in the experiments). Thus we extract the $\phi$ meson mass considering partially quenched results of the $\rho$ correlator. This is discussed in Sect. VIA.

\section{The $0^{++}$channel: $a_{0}$}

The scalar channel is difficult as usual. The mass plateau is short and varies depending on the chosen set of interpolators. The ground state mass of $a_{0}$ is very close to the lowest energy level of the (dynamical) two-particle state $\pi \eta_{2}$ (see Fig. [5), as discussed in [78, 79].

We find large effects due to partial quenching close to the dynamical point at small pion masses which are very obvious in ensemble C (see Fig. 5). The partially quenched data do not smoothly extrapolate to the dynamical point. An explanation has been offered in 65]: The partially quenched states may couple to 


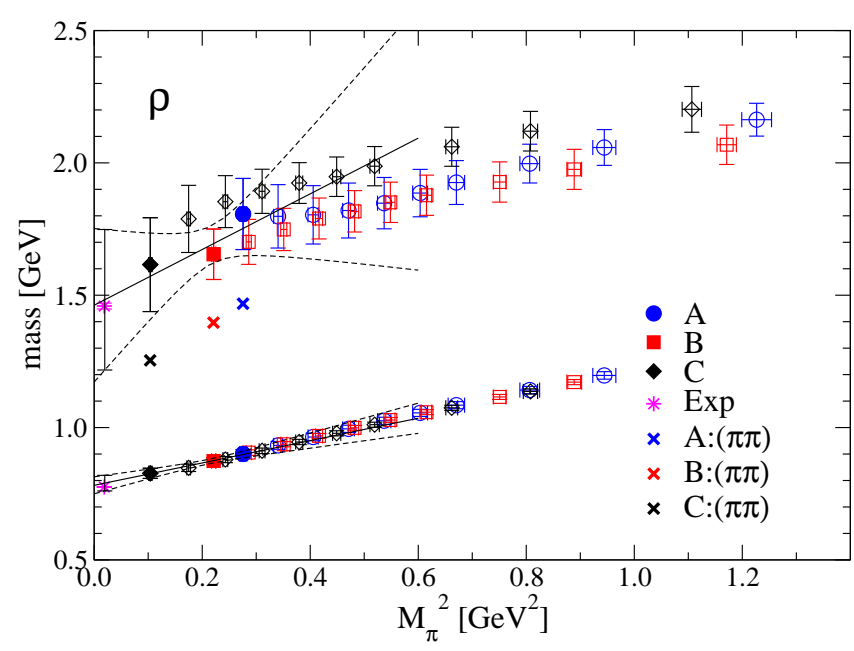

FIG. 3: Mass plot for the $1^{--}$channel $(\rho)$, ground state and first excitation. The ground state is measured using interpolators $(1,4)$, the excitation using interpolators $(7,8,13,14)$ in ensemble A, $(6,11,14)$ in B and C. The estimated energy level of the $P$ wave scattering state $\pi \pi$ lies below the first excitation in all three ensembles. The scattering state is not observed, the reason may be too weak coupling to the used interpolators. The coupling may be especially weak in case of $P$ wave scattering states. The statistical error of the 2-particle state (based on the errors of the particle masses involved) is of the magnitude of 5-10 MeV and therefore not visible in the figure.

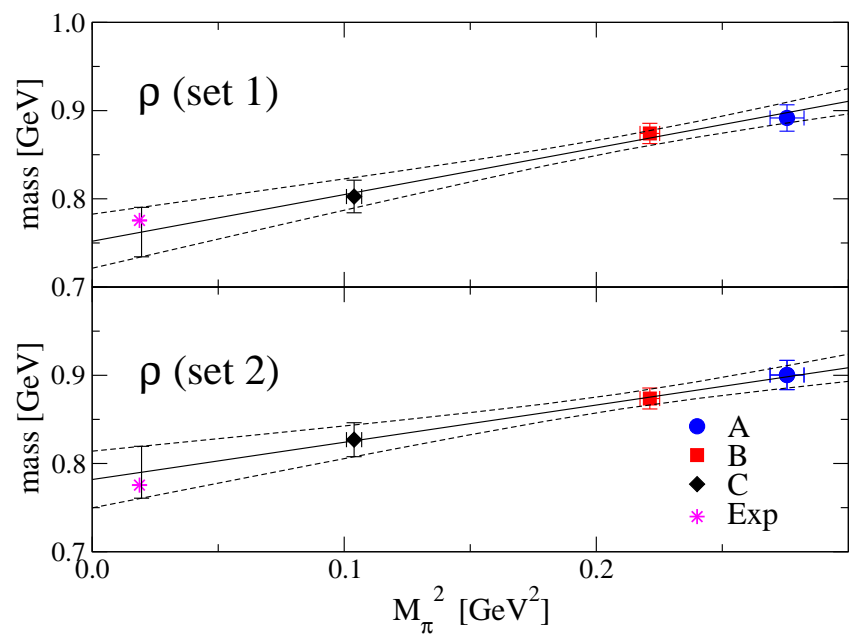

FIG. 4: Zoom in the mass plot for the $1^{--}$channel $(\rho)$, ground state. The different sets of used interpolators are checked for consistency in the ground state. Top: Interpolators $(7,8,13,14)$ in ensemble A, $(6,11,14)$ in B and C. Bottom: Interpolators $(1,4)$.

pairs of pseudoscalars (composed of valence and sea quarks), leading to unphysical contributions that cancel only in the fully dynamical case. We find that our partially quenched data are well described by the partially quenched formulae of the scattering state, and thus interpret the partially quenched data as the 2-particle state
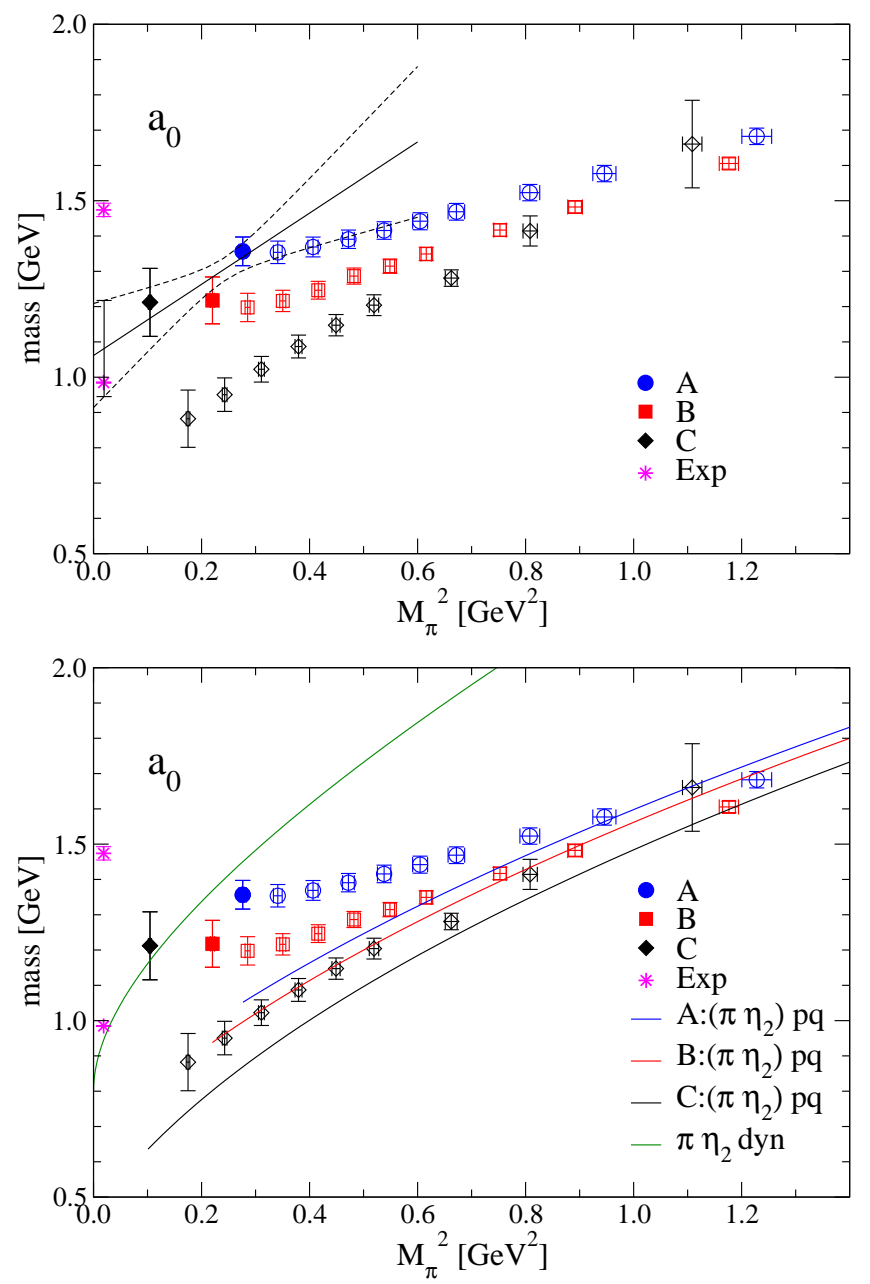

FIG. 5: Mass plot for the $0^{++}$channel. Interpolator (8) is used throughout. Top: Ground state of $a_{0}$ and chiral extrapolation relying on a 1-particle interpretation are shown. Bottom: Estimate of the two-particle state $\pi \eta_{2}$ for various parameters shown. The blue, red and black curve (online version) show a prediction of the partially quenched ("pq") scattering state $\pi \eta_{2}$ for $m_{\text {val }} \gg m_{\text {sea }}$ in ensemble A, B and C, respectively. The green curve (online version) shows an estimate of the dynamical ("dyn") scattering state $\pi \eta_{2}\left(m_{v a l}=m_{\text {sea }}\right)$. Interaction of the bound states and finite volume effects are neglected in the energy level of the two-particle state. For clarity, the corresponding statistical error is omitted in the figure. The partially quenched data suggests an interpretation in terms of the two-particle state $\pi \eta_{2}$, while no clear statement about the particle content can be made at the dynamical point.

$\pi \eta_{2}$. We stress that our results do not allow for a clear interpretation as 1- or 2-particle state at the physical point. The question of the coupling of our interpolators to the scattering states has already been discussed in Sect. IIIC3 Note that the scattering state $K \bar{K}$ cannot appear in our simulations since it involves strange quark loops.

In quenched simulations scattering states cannot con- 


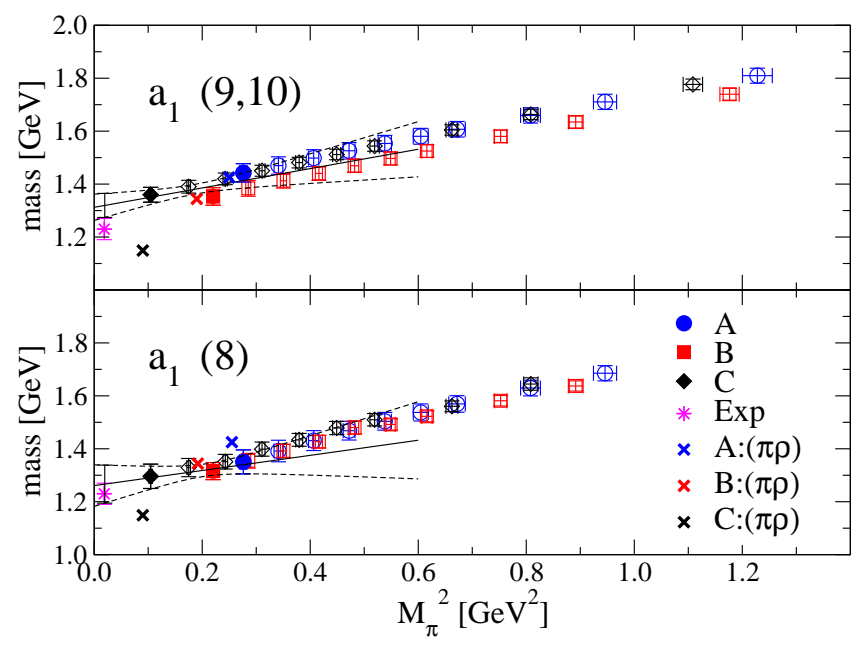

FIG. 6: Mass plot for the $1^{++}$channel $\left(a_{1}\right)$, ground state. Results using interpolators $(9,10)$ are shown in the top plot, results using interpolator (8) at the bottom. The uncertainty stemming from the choice of interpolators is discussed in the text. Note that the $a_{1}(1260)$ is a very broad resonance. The estimated energy level of the $S$ wave scattering state $\pi \rho$ is below the ground state in ensemble $\mathrm{C}$ and comparable to the ground state mass in ensemble A and B. For better identification, we display the scattering states slightly shifted to the left. We may interpret that we do not observe the scattering state, at least in ensemble C.

tribute to the signal when using 1-particle interpolators. Also, in particular in the light scalar channel, ghosts may appear and complicate the spectroscopy. A strategy to disentangle the contributions has been discussed in [42]. The ground state energy level in quenched simulations with the same action [43, 45] was extracted only at larger pion masses. There it was essentially compatible with our dynamical data of set A, extrapolating to the $a_{0}(1450)$ rather than to $a_{0}(980)$. The spectroscopy of the light scalar channel appears to benefit from sea quarks.

\section{The $1^{++}$channel: $a_{1}$}

Considering the pseudo vectors, we encounter some practical difficulties. Gaussian shaped interpolators do not yield reliable signals and derivative interpolators are needed. We obtain short plateaus even for the ground state and there appears an uncertainty associated with different choices of interpolators. We show results from two sets of interpolators to illustrate this issue (see Fig. 6). In principle, such a situation is possible if the interpolators couple to different physical states or show different discretization effects. In the absence of such effects, the results from different sets should agree within error bars and, indeed, the error bars overlap. Note that the $a_{1}(1260)$ is a very broad resonance, hence scattering states may complicate the spectroscopy. Based on experiments one could expect an $S$ wave $\pi \rho$ energy level

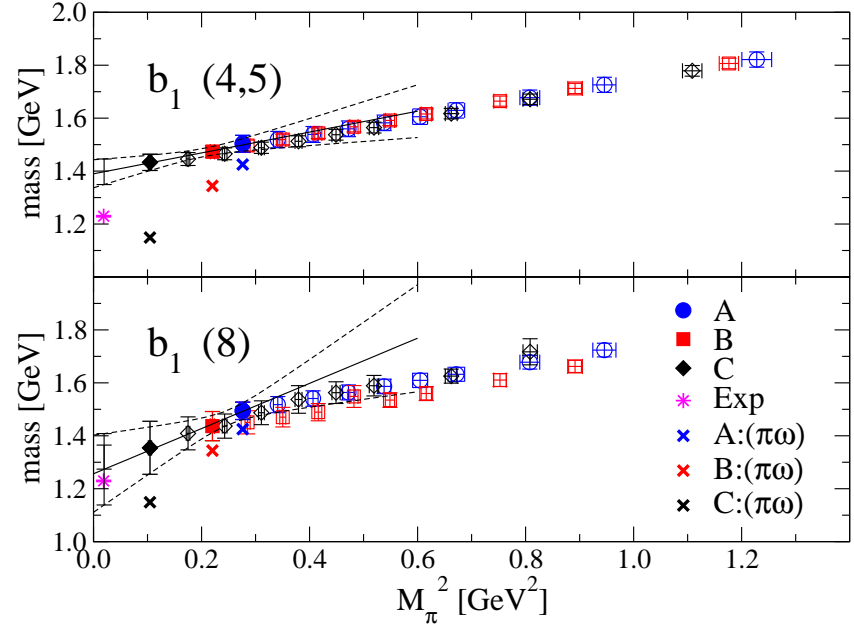

FIG. 7: Mass plot for the $1^{+-}$channel $\left(b_{1}\right)$, ground state. Results using interpolators $(4,5)$ are shown in the top plot, results using interpolator (8) at the bottom. The uncertainty stemming from the choice of interpolators is discussed in the text. The energy level of the $S$ wave scattering state $\pi \omega$ is estimated using the approximation $m_{\omega}=m_{\rho}$. For better identification, we display the scattering states slightly shifted to the left. The energy level is below the ground state, in particular in ensemble C.

below the $a_{1}$ level at least for ensemble $\mathrm{C}$, which we do not observe.

The energy levels of quenched simulations using the same action [45] appear a bit high. However, they are compatible with the results presented in this work if one considers the uncertainty associated with different choices of interpolators.

\section{The $1^{+-}$channel: $b_{1}$}

Here the situation is similar to the $1^{++}$channel. The usage of derivative interpolators is mandatory, nevertheless weak plateaus are obtained and one finds an uncertainty which is even larger than in the $a_{1}$ channel. Again, we show results from two combinations of interpolators (see Fig. 7). Using interpolators $(4,5)$ one gets a result rather far from the experimental $b_{1}(1235)$, while the result of interpolator (8) is compatible with experiment within a large error bar. Both results are consistent within error bars. The energy level of the $S$ wave scattering state $\pi \omega$ is estimated using the approximation $m_{\omega}=m_{\rho}$. However, this rough estimate does not allow for any precise statement about the particle content of the measured state.

\section{The $2^{++}$channel: $a_{2}$}

In the spin 2 channels we encounter for the first time the situation to have orthogonal irreducible representa- 

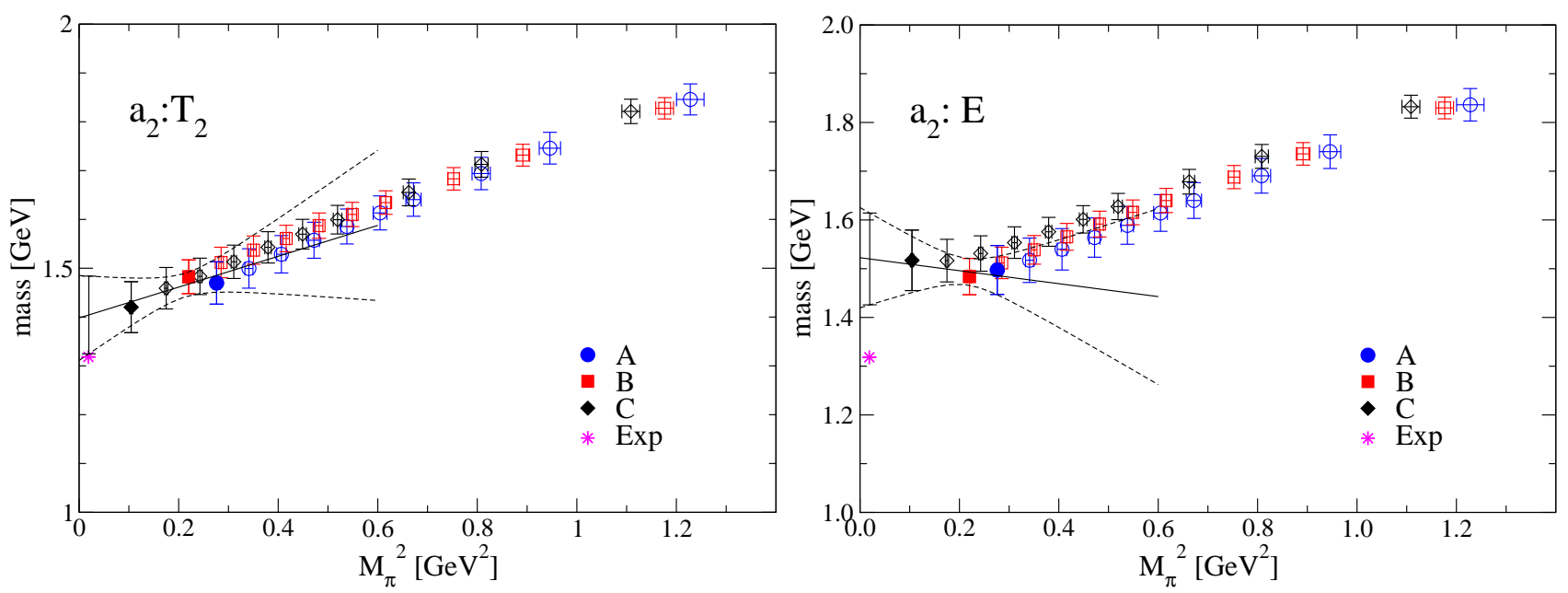

FIG. 8: Mass plot for the $2^{++}$channel $\left(a_{2}\right)$, ground state. Lhs: Irrep $\mathrm{T}_{2}$, using interpolators $(1,2,3,4)$ in ensemble A and B, $(2,3)$ in C. Rhs: Irrep E, interpolators $(1,2,5)$ are used throughout. The result of ensemble C likely suffers from small statistics, leading to a negative slope of the chiral fit.

tions (irreps) on the lattice, which couple to the same spin state in the continuum limit. Hence we are able to compare results from these different irreps. Fig. 8 shows the mass of $2^{++}$in irrep $\mathrm{T}_{2}$ and $\mathrm{E}$. In both representations using only one interpolator does not yield a reliable signal and employing the variational method is necessary. The resulting mass of $\mathrm{T}_{2}$ agrees with the experimental $a_{2}(1320)$ within one $\sigma$. The resulting mass of $\mathrm{E}$, however, comes out too high, where the reason seems to be a large mass of ensemble $\mathrm{C}$, leading to a negative slope of a linear fit.

\section{The $2^{--}$channel: $\rho_{2}$}

Unfortunately, in the irrep $\mathrm{T}_{2}$ we can extract a mass only in two of the 3 ensembles (see Fig. 9). Enlarged statistics will be necessary in ensemble $\mathrm{A}$ in order to observe a reliable mass plateau. Also in the other ensembles and in the irrep E the mass plateaus are short and the fit ranges are partly motivated by the clearer plateaus of partially quenched mass results. Nevertheless, the mass obtained is consistent with the experimental $\rho_{2}(1940)$ (see Fig. 9). An estimate of the scattering states appearing in this channel is shown in the figure. It seems possible that the measured state involves contributions from the $S$ wave scattering state $\pi a_{2}$, however, the rough estimate does not allow for any clear statement.

Note that in the continuum limit the irrep $\mathrm{T}_{2}$ couples also to spin 3 and irrep $\mathrm{E}$ to spin 4 states. Hence a signal of $\rho_{3}(1690)$ could be seen in $T_{2}$, but not in E. However, the interpolators used have naive continuum limit of spin 2 , which we consider to be the reason for the missing of a signal of $\rho_{3}(1690)$. We stress that also in this channel higher statistics is necessary for a reliable extrapolation to the physical point.

\section{The $2^{-+}$channel: $\pi_{2}$}

Here we encounter rather large statistical errors and additional uncertainty associated with different sets of interpolators (see Fig. 10.) Nevertheless, all results are consistent within $2 \sigma$. Due to the large error bar, we find the linear chiral extrapolation compatible with both, the $\pi_{2}(1670)$ and the $\pi_{2}(1880)$. As in the other spin 2 channels, the results would benefit from higher statistics.

\section{Exotics}

So-called exotic states have quantum numbers which cannot be constructed using a naive quark model. Most of the known exotic particles are found above $2 \mathrm{GeV}$, but also some lower ones are known, e.g. $\pi_{1}(1400)$ and $\pi_{1}(1600)$ in the $1^{-+}$channel. We implemented interpolators with exotic quantum numbers using derivative sources. Unfortunately, the obtained data are very noisy and we found it impossible to perform a fit of the effective masses. Since we observe a very weak signal in the $1^{-+}$channel, we hope that a fit can be done when larger statistics becomes available.

\section{B. Baryons with light quarks}

Our baryon interpolators are built from Gaussian sources only (no derivative sources) and can be found in Appendix A. All interpolators are projected to definite parity in each channel. 

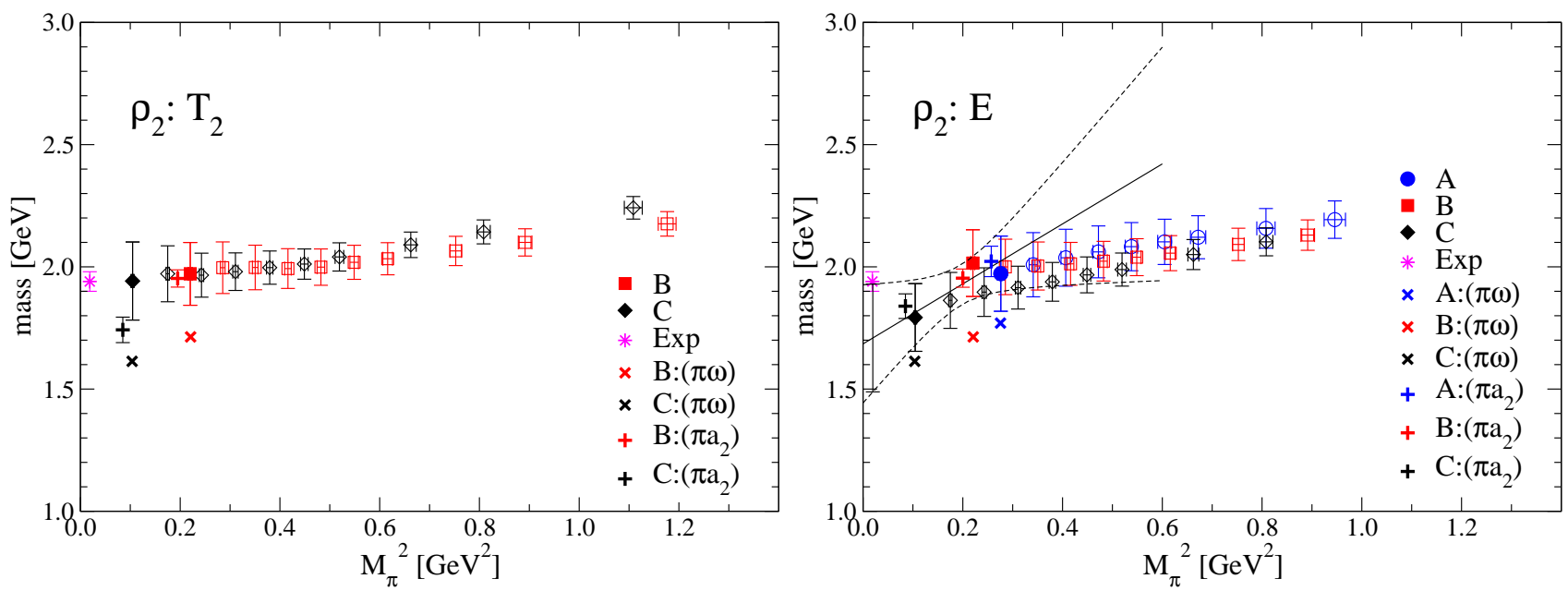

FIG. 9: Mass plot for the $2^{--}$channel $\left(\rho_{2}\right)$, ground state. Lhs: irrep $\mathrm{T}_{2}$, interpolator (1) is used in ensemble B and C. Rhs: irrep E, interpolator (1) is used throughout. The energy level of the $P$ wave scattering state $\pi \omega$ is estimated using the approximation $m_{\omega}=m_{\rho}$. It is found to be below the ground state in all three ensembles. The higher energy level of the $S$ wave scattering state $\pi a_{2}$ is in better agreement with the observed states. However, the estimate does not allow to identify the particle content of the measured state.
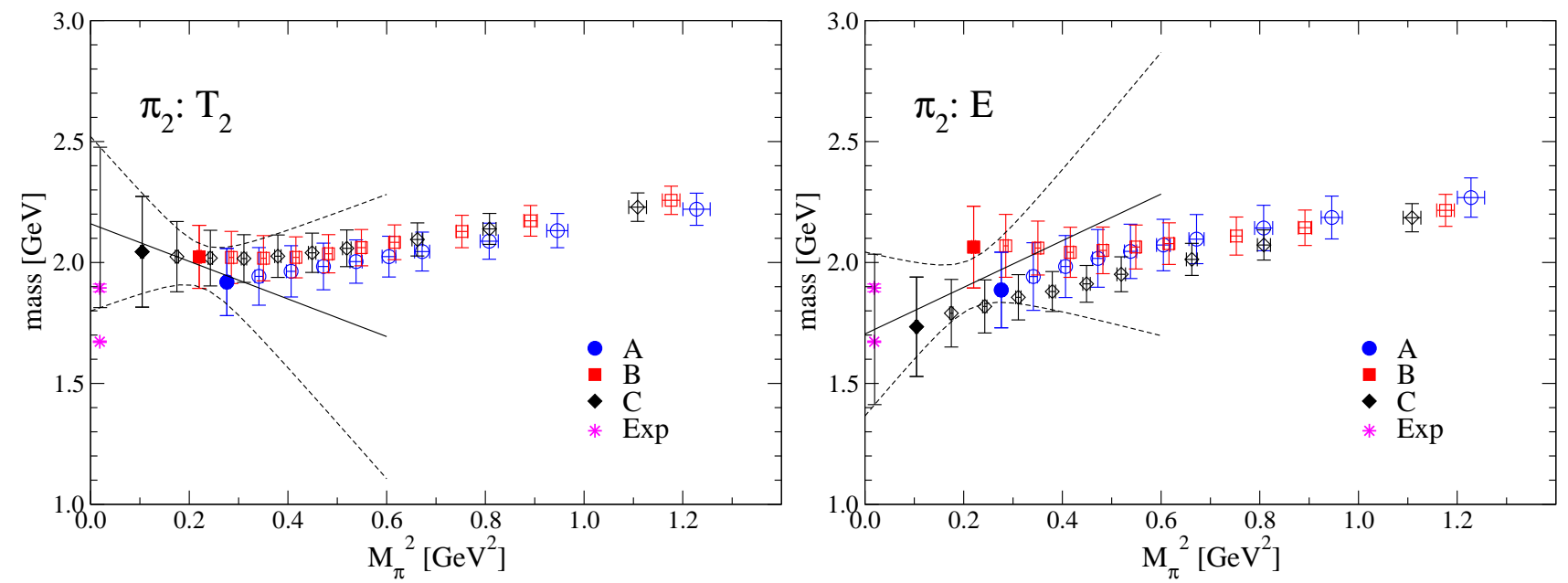

FIG. 10: Mass plot for the $2^{-+}$channel $\left(\pi_{2}\right)$, ground state. Lhs: Irrep $\mathrm{T}_{2}$, interpolator $(1)$ is used throughout. Rhs: Irrep E, interpolators $(1,2)$ are used throughout.

\section{Nucleon positive parity}

The nucleon positive parity ground state can be extracted to good precision as usual (see Fig. 11). A conventional fit linear in the pion mass squared yields a nucleon mass a bit higher than the experimental value. In [80, 81] it was found that a simple fit linear in the pion mass of the nucleon positive parity ground state agrees well with experiment. Indeed such an extrapolation agrees well with experiment also in our case. However, a reliable clear distinction would only be possible using more data from dynamical simulations and higher statistics. Therefore, we stick to the expectation of the analytic behavior being close to the pion mass squared as suggested from ChPT and thus we quote the corresponding fit linear in the pion mass squared in the conclusions.

The first excitation would be compatible with the energy level of the $P$ wave scattering state $\pi N$. However, following the arguments of Sec. III C3 we believe to see an almost pure 1-particle state.

The first nucleon excitation, the so-called Roper, comes out several hundred $\mathrm{MeV}$ too high in dynamical simulations [18, 31, 32]. There are several possible explanations for this. On one hand, whereas the negative parity ground state baryons are orbital excitations (according to the quark model), the Roper is a radial excitation. Thus its size may be substantially larger than that of the nucleon and affected by squeezing due to the spatial volume size. Another reason may be a strong in- 

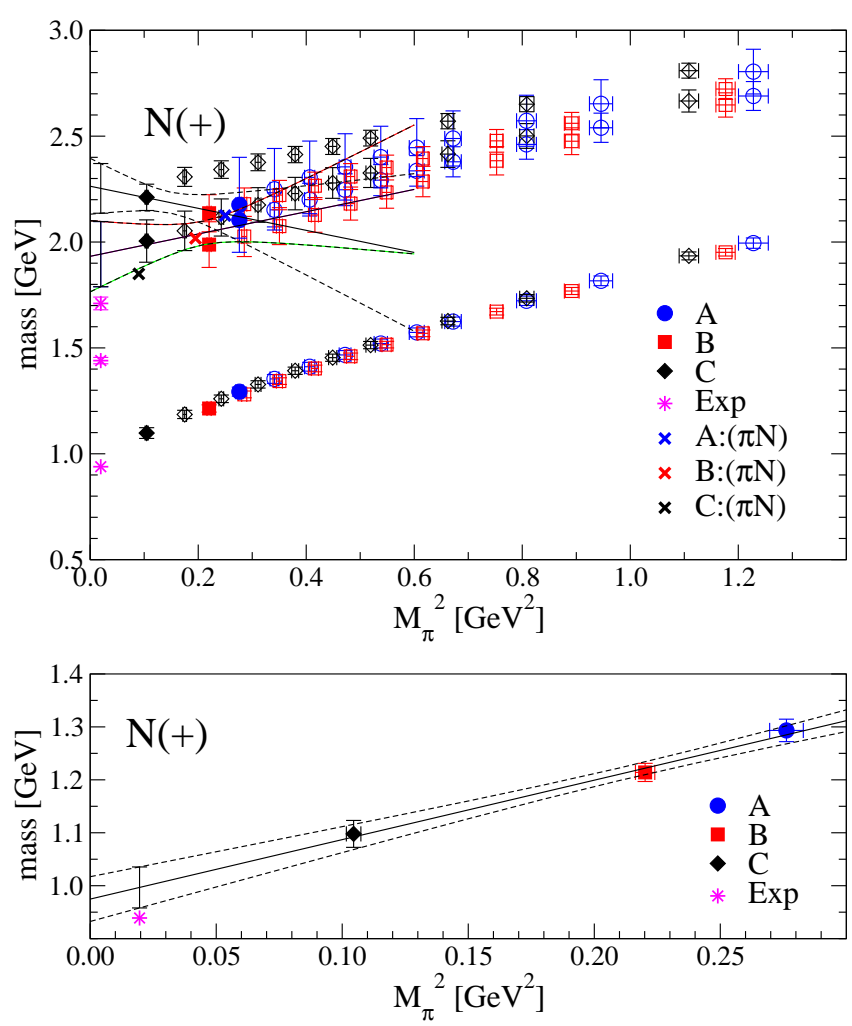

FIG. 11: Mass plot for the nucleon positive parity channel. Top: Results for ground state and two excitations. The energy level of the $P$ wave scattering state $\pi N$ is close to the first excitation in all three ensembles. For clarity, we display the scattering states slightly shifted to the left. Bottom: Fit to the ground state mass of the nucleon positive parity channel. Interpolators $(1,2,13,14)$ are used in ensemble A, $(1,2,4,14,15,18)$ in B and C.

fluence from the $\pi N$ channel, which may not be properly represented by our set of interpolators (cf., the discussion in Sect. III C 3). It may be necessary to explicitly include such meson-nucleon interpolators, which then, however, poses the technical challenge of backtracking quark loops.

Comparing to the corresponding quenched simulations [44], we do not observe a significant qualitative difference of the results in the nucleon positive parity channel. Our data are also in agreement with quenched and dynamical results from other groups (e.g., 25, 27, 32]).

\section{Nucleon negative parity}

In general, the signal is rather noisy in negative parity baryon channels. In the case of the nucleon, we also find that the backwards-running positive parity nucleon (and possibly also back-to-back scattering states [82]) limits the fit window to the interval $3 \leq t \leq 7$. However, we even find a signal of the first excitation, which is close to the ground state, both being compatible with experimental data (see Fig. 12). We find that all our negative

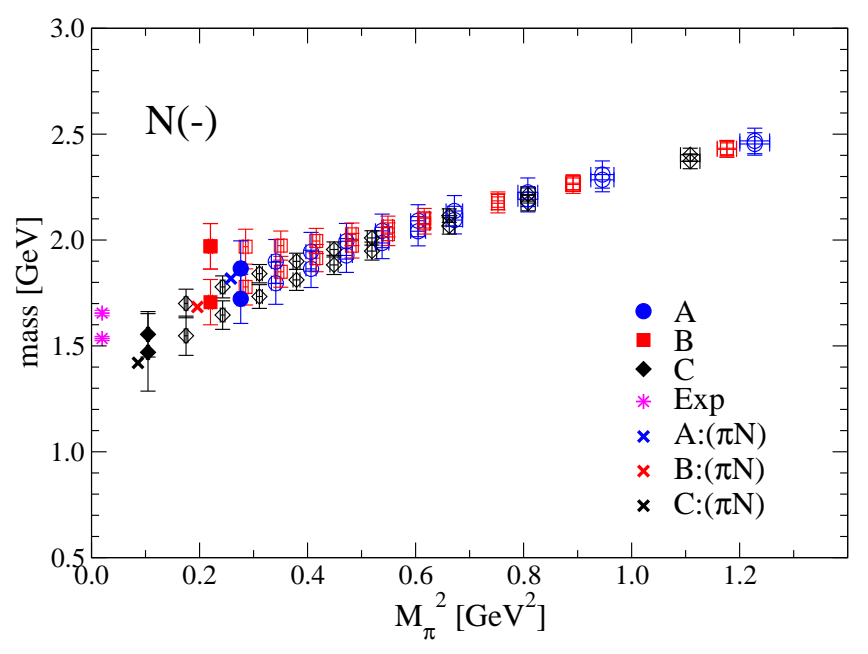

FIG. 12: Mass plot for the nucleon negative parity channel, ground state and first excitation. The energy level of the $S$ wave scattering state $\pi N$ is close to the first excitation in A and close to the ground state in B and C. Naively, this could be interpreted as a hint for a level crossing of the 1particle ground state and the scattering state. Also the low results in case of ensemble $\mathrm{C}$ suggest such an interpretation. However, the eigenvectors contradict this picture (see Fig. 13). Interpolators $(2,7,9)$ are used in ensemble $\mathrm{A},(1,3,7,8,9)$ in $\mathrm{B}$ and $(1,7,8,9)$ in C. For clarity, we display the scattering states slightly shifted to the left and omit the chiral extrapolation in the Figure.

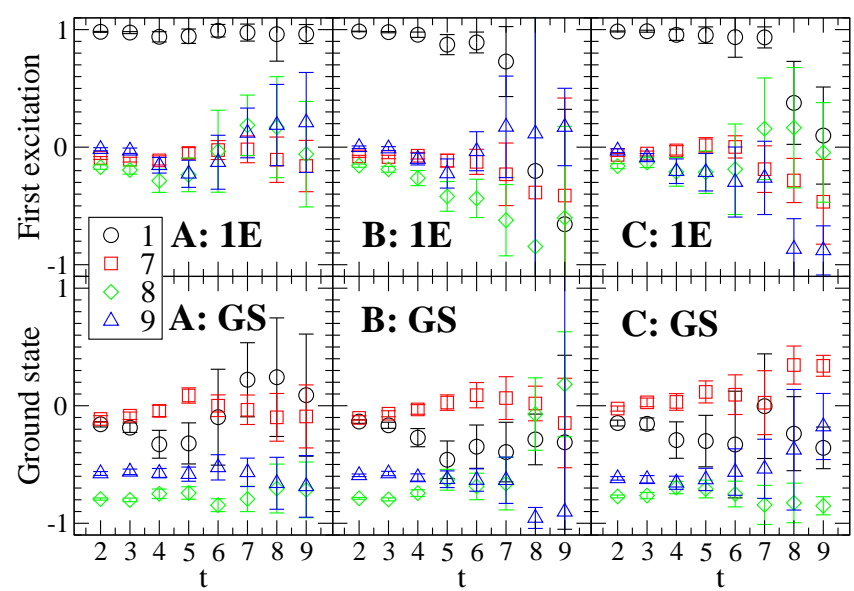

FIG. 13: Normalized eigenvectors of the nucleon negative parity channel, ground state and first excitation at the dynamical point. To allow for a direct comparison, the same set of interpolators $(1,7,8,9)$ is used in all three ensembles A, B and C. The corresponding fits of the eigenvalues are performed in the time range $4 \leq t \leq 7$. In all three ensembles, the ground state is dominated by the second Dirac structure $\left(\chi_{2}\right)$, while the first excitation is an almost pure $\chi_{1}$ state (see Table $\mathrm{X}$. One may conclude that no level crossing of the lowest two states is observed for pion masses in the range between 320 and $520 \mathrm{MeV}$. Note that in order to extract masses, different sets are used in A and B, which yield more stable plateaus. 
parity baryon ground states come out somewhat too low.

In nature, the $S$ wave state $\pi N$ (assuming free particles) is below the one-particle ground state in the nucleon negative parity channel. This may also be true at slightly larger pion masses, e.g., in ensemble C. That would explain the low results in $\mathrm{C}$ and thus the low chiral extrapolation as well. At large pion masses, the scattering state becomes heavier than the 1-particle ground state and one expects a level crossing to take place in between. Indeed, our results on masses in the three ensembles are compatible with such a picture.

However, in contrast to the scalar channels, we can extract useful information from the eigenvectors in the nucleon and sigma negative parity channels. The reason is that several interpolators are needed for a good signal and that two states are observed. We find that in all three ensembles the ground state is dominated by the second Dirac structure $\left(\chi_{2}\right)$, while the first excitation is an almost pure $\chi_{1}$ state (see Fig. 13 and Table $\mathrm{X}$ ). This property is seen even more clearly for partially quenched points, where the plateau is more stable. One may conclude that no level crossing of the lowest two states is observed for pion masses in the range of 320 to $520 \mathrm{MeV}$.

Another hint comes from the comparison with old quenched results using the same action [44]. Using only 3 -quark nucleon interpolators, no scattering states can appear in quenched simulations. Hence, the eigenvectors in the quenched simulations can clearly be identified with 1-particle states. We stress that in the quenched approximation ghosts appear at low pion masses, thus a reliable comparison to dynamical simulation can be done only at large pion masses. We assume the pions of ensemble A to be heavy enough to allow for a comparison with quenched simulations. By subsequent comparison of dynamical simulations at decreasing pion masses, in principle conclusions down to physical pion masses are possible. Unfortunately, direct comparison of the quenched eigenvectors with our dynamical ones is impossible, since the corresponding interpolators differ slightly (e.g., in the width of the Gaussian source). However, qualitatively, we find the same Dirac structure content of the two lowest states. From that, one may conclude that both our lowest two states are 1-particle states, which is also suggested from the missing level crossing of the eigenvectors.

So, while the extracted mass values slightly favor the interpretation of a scattering state in ensemble $\mathrm{C}$, the eigenvectors tell a different story. Since the line of arguments based on the eigenvectors seems to be more reliable, we believe to see almost pure 1-particle states and quote the corresponding chiral extrapolation in the summary.

In the quenched simulation with the CI-action [44], nucleon negative parity masses have been extracted only at larger pion masses. The bending down at low pion masses observed in the present work can thus not be compared directly.

Note that in order to extract masses, different sets are used in A and B than the ones shown in Fig. 13. In en-

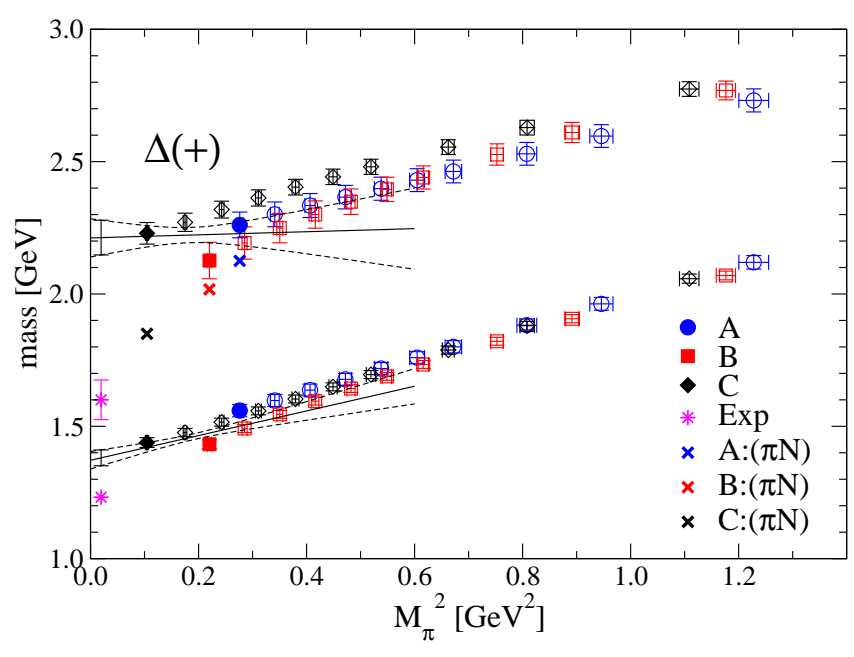

FIG. 14: Mass plot for the $\Delta$ positive parity channel, ground state and first excitation. The energy level of the $P$ wave scattering state $\pi N$ is close to the first excitation in $\mathrm{A}$ and $\mathrm{B}$, but clearly separated from any measured state in C. Interpolators $(1,2,3)$ are used in ensemble $\mathrm{A}$ and $\mathrm{C},(2,4,6)$ in B.

semble $\mathrm{C}$, the fit range is motivated by tracing of plateaus of partially quenched results.

\section{3. $\Delta$ positive parity}

In the $\Delta$ positive parity channel, we find the masses being too high, in particular the first excitation (see Fig. 14), although they are lower than in the quenched analysis [4]. Since the statistical error is fairly small, systematic errors and finite volume effects seem to be responsible. As will be discussed below, this channel - for large values of the valence quark mass - is used to identify the strange quark mass parameter. Analogous to other $P$ wave scattering states, $\pi N$ seems to be missing in our simulation.

Quenched results using the CI-action 44] have shown a similar systematic upwards shift of the masses. Our $\Delta$ positive parity ground state is compatible with other groups (e.g., 32]). However, there the first excitation is fairly close to the ground state, which we do not observe here. A possible reason could be the larger basis of interpolator used in that work. Hence, we cannot exclude possible systematic errors associated with the choice of interpolators in this channel.

\section{4. $\Delta$ negative parity}

There is a clear signal found of the $\Delta$ negative parity channel ground state (see Fig. 15). The energy level of the $S$ wave scattering state $\pi N$ is below the ground state in all three ensembles. The results seem to be in better agreement with an interpretation in terms of a 1-particle 


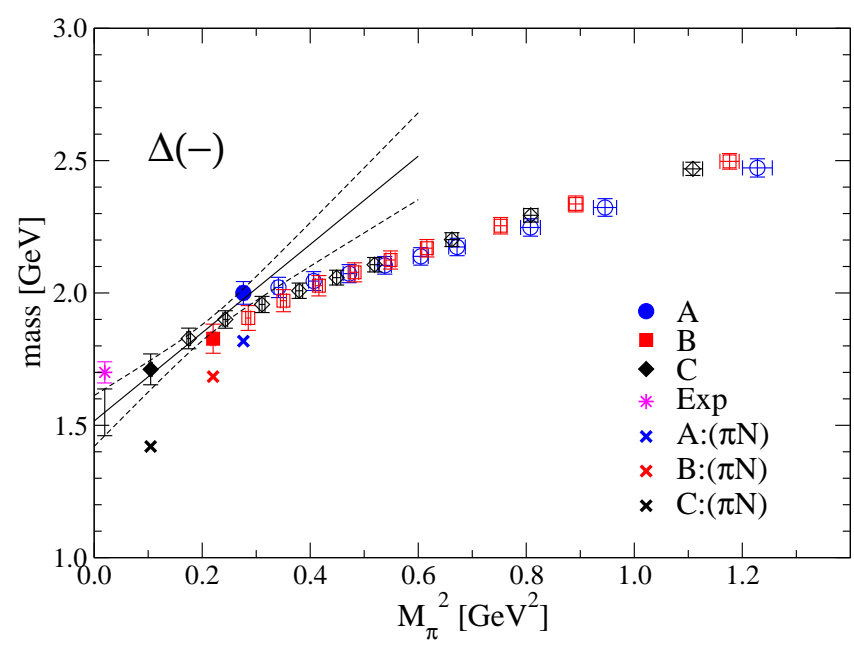

FIG. 15: Mass plot for the $\Delta$ negative parity channel, ground state. The energy level of the $S$ wave scattering state $\pi N$ is below the ground state in all three ensembles. We display the scattering states slightly shifted to the left. Interpolator (3) is used in ensemble A, $(2,3)$ in B and C.

state.

Quenched results of 44] have been extracted only at rather large pion masses, making a comparison to present results uninstructive.

\section{RESULTS: STRANGE HADRONS}

We extract the strange quark mass parameter by identification of a partially quenched $\Delta$ (i.e., valence quark of larger mass) with $\Omega(1670)$, as described in Sect. VIA The interpolators used for strange meson spectroscopy are listed in Appendix $\mathrm{A}$. Since $C$-parity is an exact symmetry for mesons only in the case of mass-degenerate quarks, we consider interpolators without projection to definite $C$-parity for the strange mesons. This means that we do not perform a symmetrization of the interpolators in the strange meson sector. Our strange meson correlator calculation lacks cross correlations between interpolators of different $C$-parity quantum numbers in the limit of degenerate quark masses. Therefore, when analyzing strange mesons, we have to restrict ourselves to subsets of interpolators sharing the same $J^{P C}$ quantum numbers in the limit of degenerate quark masses.

In all plots shown for strange hadrons, the full symbol represents a hadron where the valence strange quark mass is determined from the $\Omega(1670)$ and the light quark has the mass of the dynamical quarks. The open symbols denote partially quenched data, where only the light valence quark mass varies. We thus neglect the effect of a dynamical strange quark, motivated by the dominance of light quark loops over strange quark loops.

A quantum field theory, where the sea quark masses do not agree with the valence quark masses, is "sick", as can be seen, e.g., by the appearance of ghosts which

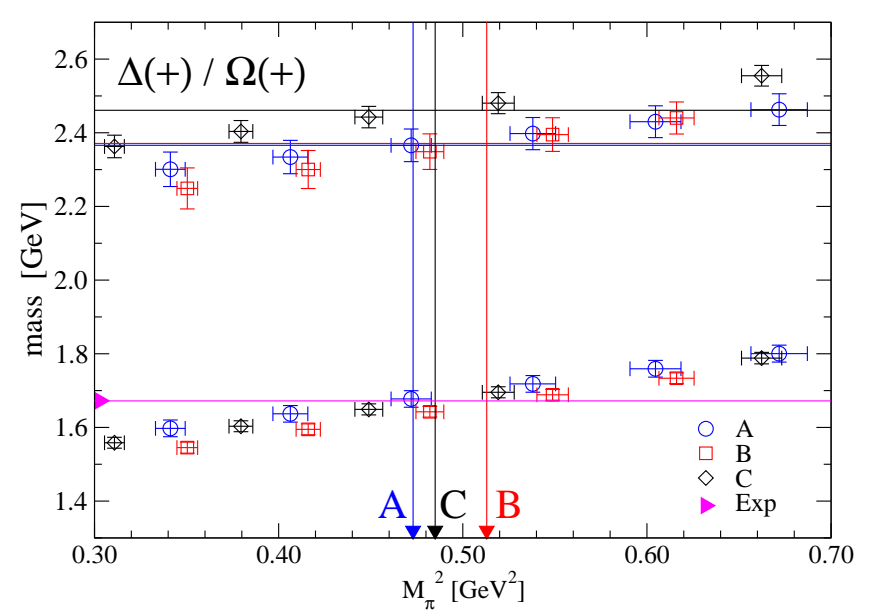

FIG. 16: Extracting the strange quark mass parameter by identifying a partially quenched $\Delta$ with $\Omega(1670)$. The lowest (magenta) horizontal line represents the $\Omega(1670)$. Crossing this horizontal line with the partially quenched $\Delta$ mass curves of ensembles $\mathrm{A}, \mathrm{B}$ and $\mathrm{C}$ defines the strange quark mass parameter of the three ensembles, illustrated by the three vertical lines in the corresponding colors. The resulting bare quark mass parameters are $-0.020,-0.015$ and -0.022 , for ensembles A, B and C, respectively. The calculation for the excited $\Omega$ around $2400 \mathrm{MeV}$ is expected to be too high due to finite volume effects.

violate the spin-statistic theorem. As discussed in [65], the correlators are strongly affected by partial quenching if the valence quarks are lighter than the sea quarks. In our simulations we consider only valence quarks heavier than or equal to the sea quarks.

Clearly, it would be desirable to include strange sea quarks in the simulation.

\section{A. Setting the strange quark mass}

The $\Omega(1670)$ consists of three strange valence quarks and shows weak dependence on the light quark masses. $\Omega$ and $\Delta$ share the same $J^{P}$ quantum numbers, they differ only in their flavor content. Therefore, we use our partially quenched results in the $\Delta$ positive parity channel to identify the strange quark mass parameter for our ensembles A, B and C (see Fig. 16). We decided to choose strange quark mass parameters which fit the experimental $\Omega$ within our error bars, allowing only for parameter values in discrete steps of 0.05 additive to the sea quark mass. In case of ensemble A the obtained mass parameter perfectly fits one of the already available quark propagators. In case of ensembles $\mathrm{B}$ and $\mathrm{C}$, we decided to recompute the quark propagator at the strange quark mass instead of interpolating between the two adjacent values.

Another possibility to extract the strange quark mass parameter would be to apply the same recipe to a partially quenched $\phi$ in the $1^{--}$channel. The decay channels 


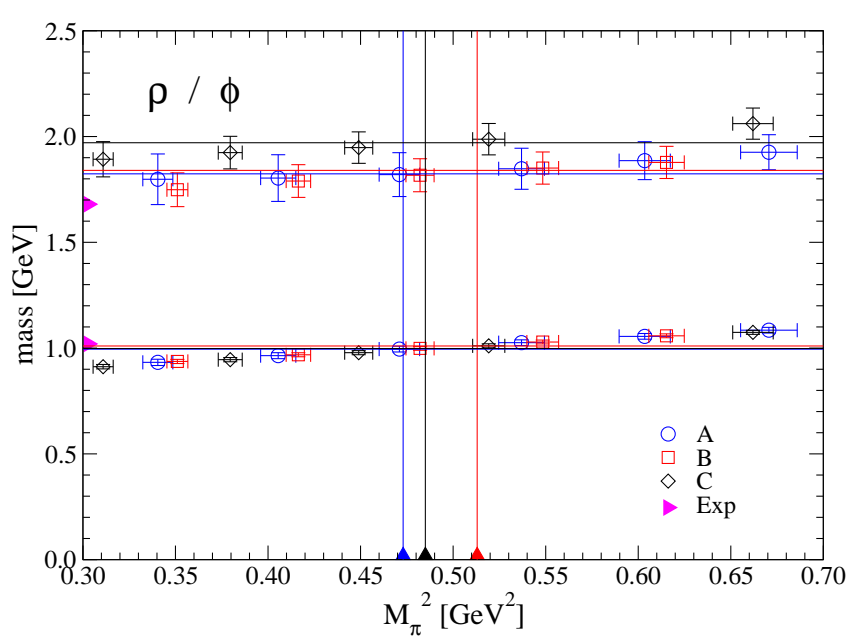

FIG. 17: Cross-check of the obtained strange quark mass parameter: The partially quenched $\phi$ from the ground state of the $1^{--}$channel fits the experimental $\phi(1020)$ very nicely. The result for the excited $\phi$ is higher than the experimental value, the deviation may be due to the neglected disconnected diagrams or simply due to the weak signal.

of $\phi(1020)$ suggest that it is dominated by its $(s \bar{s})$ flavor content. We use $\phi$ as a cross-check for the strange quark mass obtained via $\Omega(1670)$. The result fits the experimental $\phi(1020)$ very nicely (see Fig. 17), indicating that our approach is consistent. The excited state of $\Omega$ is assumed to suffer from finite size effects. The signal of the excitation of $\phi$ suffers from neglected disconnected diagrams and poor statistics. Thus these two levels are not appropriate for further checks of the strange quark mass. However, the ground state levels of $\Sigma$ and $\Xi$ positive parity may be regarded as additional affirmative cross-checks (see Figs. 25] and 27).

Even in the case of the $\Omega$, one could expect finite size effects, since they show up in the $\Delta$ positive parity channel for both, ground and excited state. However, using heavier quarks, finite size effects are expected to become less and less important in the ground state. The crosscheck using $\phi$ verifies this expectation.

\section{B. Mesons with strange quarks}

\section{The $0^{-}$channel: $K$}

In the strange meson channel $0^{-}$we find a very accurate determination of the $K$ ground state, and a fairly reliable result of the first excitation which is compatible with experiment (see Fig. 18). Comparing to the pion channel, we find that the signal improves in case of a heavier valence quark, as expected.

Comparing to the corresponding quenched simulations [43], we do not observe a qualitative difference.

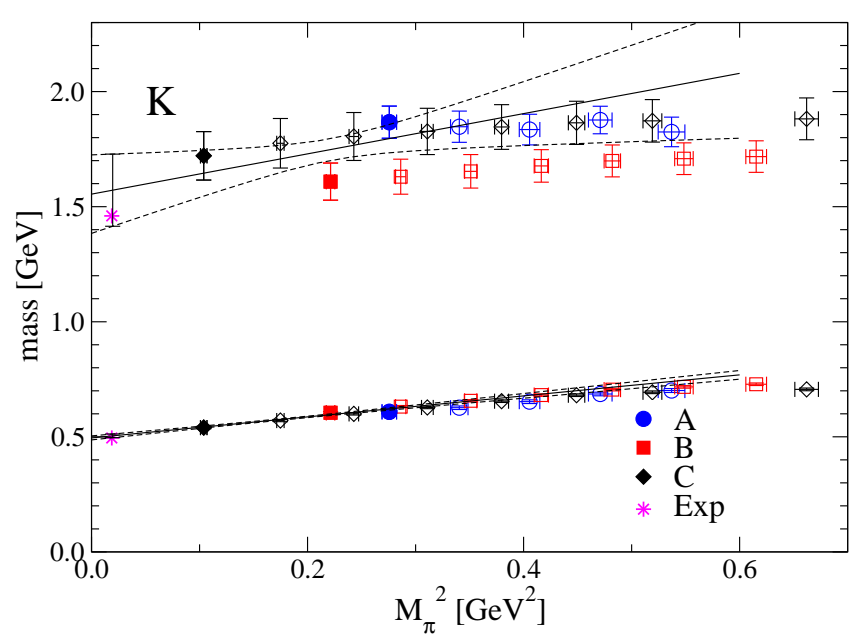

FIG. 18: Mass plot for the $0^{-}$channel $(K)$, ground state and first excitation. Ground state measured using interpolator (4), excitation measured using $(4,8,11,14,17)$ in A, $(4,7,11,14,17)$ in B and C. Note that the strange meson interpolators are not symmetrized (see Appendix $\mathbb{A}$ ). The $P$ wave scattering state $\pi K^{*}$ is assumed to be suppressed and therefore not indicated, since at the used simulation parameters its energy is comparable to the one of the first excitation.

\section{The $0^{+}$channel: $K_{0}^{*}$}

The strange scalar channel is peculiar similar to the light scalar channel. Furthermore, the lowest experimental state in this channel, $K_{0}^{*}(800)$ or $\kappa$, is not established and a very broad resonance with a width of more than $80 \%$ of its mass. It is thus not clear whether this state is expected to be observed in our simulation. The lowest established resonance listed by the Particle Data Group is $K_{0}^{*}(1430)$. At the parameters we use, an appearance of a low-lying scattering state $\pi K$ is possible. The lowest momentum two-particle state (assuming free particles) is indicated in the figure, together with the result and the experimental $K_{0}^{*}(800)$ and $K_{0}^{*}(1430)$ (see Fig. 19). The result is compatible with both, the resonance $K_{0}^{*}(800)$ and the scattering state. Hence we cannot definitely exclude either of the possibilities. Comparison to the results of the light scalar channel $0^{++}\left(a_{0}\right)$ may suggest that the scattering state contributes at least at partially quenched points. Higher statistics and another volume would be desirable in order for a more clear distinction. If we use, e.g., interpolators $(12,13)$, the eigenvectors look very similar for different valence light quark masses, which means that the state we extract remains roughly the same over the range of partial quenching we investigate. This can be interpreted as an indication that the level crossing of a scattering state and the resonance, if it exists, is not located in the mass range of our investigation. 

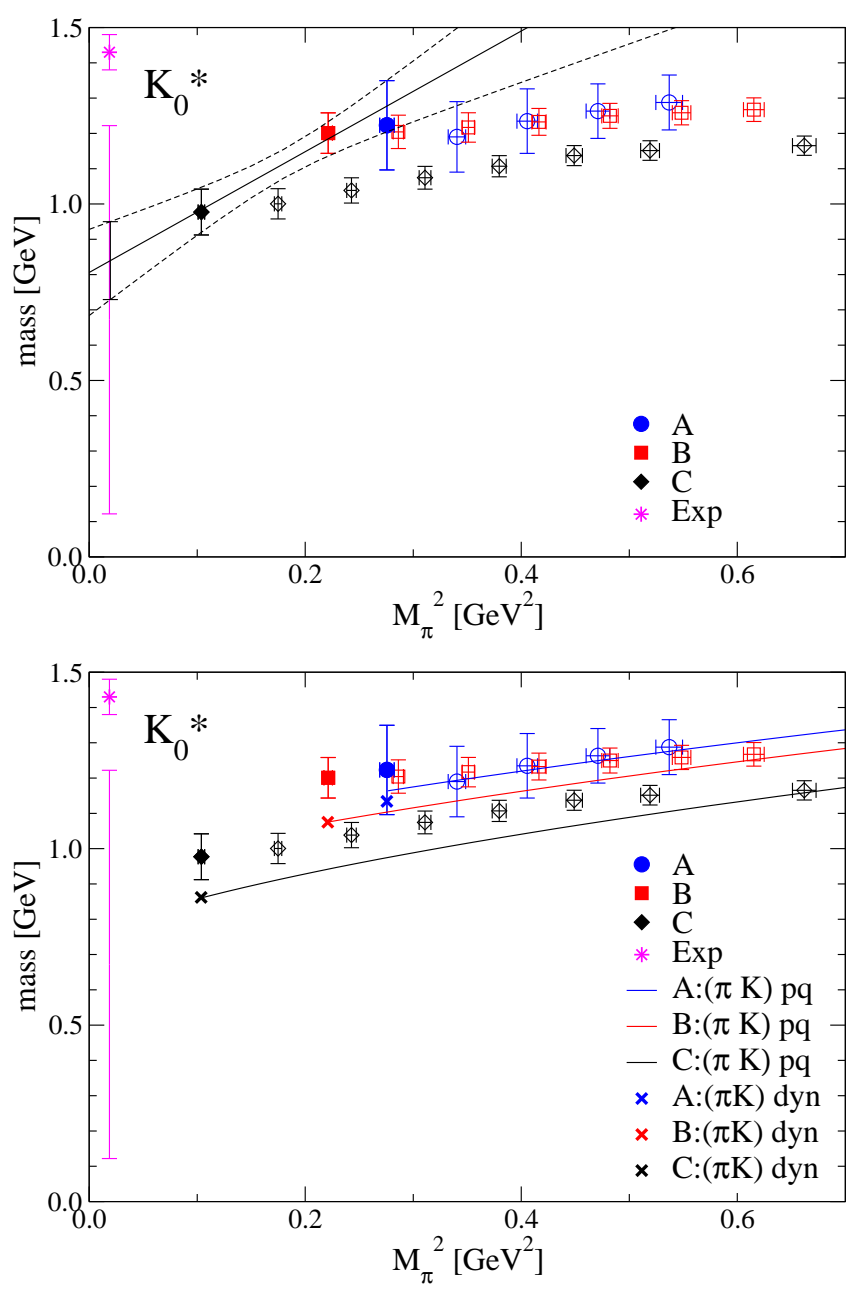

FIG. 19: Mass plot for the $0^{+}$channel, measured with interpolator 13 . Since $K_{0}^{*}(800)$ is a very broad resonance, thus we show its experimental width only. Top: Ground state and chiral extrapolation according to a 1-particle interpretation $\left(K_{0}^{*}\right)$ are shown. Bottom: The crosses represent an estimate of the dynamical $S$ wave 2-particle state $\pi K$. The blue, red and black curve (online version) show a prediction of the corresponding partially quenched ("pq") state $\pi K$ for $m_{\text {val }} \gg m_{\text {sea }}$ in ensemble A, B and C, respectively. Since there are no strong effects from partial quenching in this channel, the partially quenched prediction almost hits the dynamical one. Note that in the partially quenched case only the light valence quark varies and the strange valence quark mass is held fixed. Thus, considering the scattering state, the partially quenched pion mass varies, while the $K$ mass is constant along the partial quenching. The results do not allow for a clear statement about the particle content of the state.

\section{The $1^{-}$channel: $K^{*}$}

We obtain an accurate result of the ground state $K^{*}(892)$ and a signal of an excited state (see Fig. 20). Interestingly, the excitation fits the $K^{*}(1680)$ way better than the $K^{*}(1410)$. Compared to the light meson sector, mixing of $J^{P+}$ and $J^{P-}$ interpolators can ap-

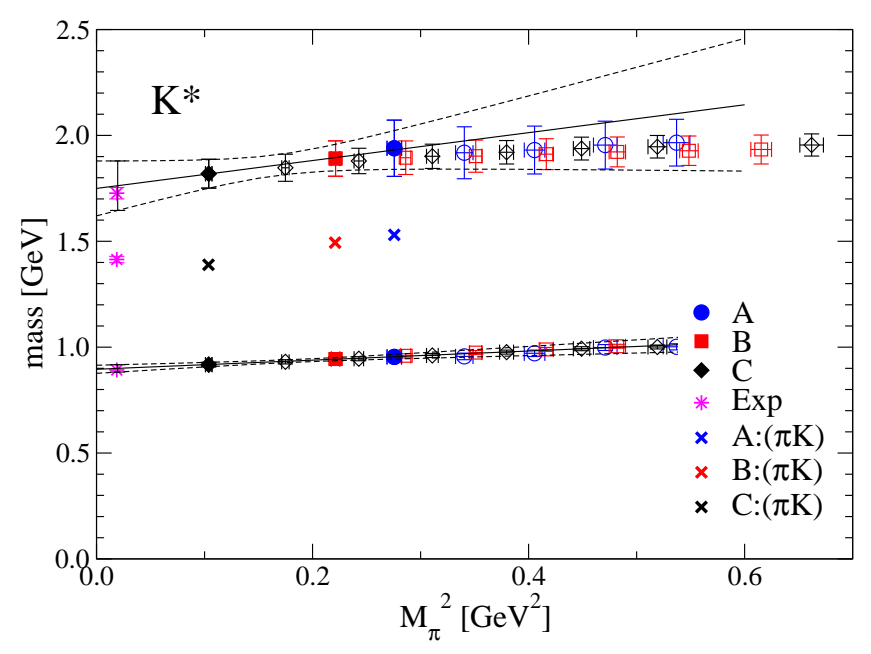

FIG. 20: Mass plot for the $1^{-}$channel $\left(K^{*}\right)$, ground state and excitation. Ground state measured using interpolators $(1,5)$, excitation measured using $(1,5,17,18,19)$. The energy level of the $P$ wave scattering state $\pi K$ is well separated from any observed state in all three ensembles.

pear in the strange meson sector, which is expected to shift the energy levels. This effect is due to the missing isospin symmetry when the involved quarks are not mass-degenerate and thus it grows when increasing the mass difference between the light and the strange quark. However, $1^{-+}$interpolators are exotic, hence it is not clear how much they contribute to the final state. It is possible that our interpolators do not show overlap with the $K^{*}(1410)$, because we restrict ourselves to the non-symmetrized interpolators corresponding to the light meson channel $1^{--}$. Another possible reason would be a tetraquark dominance of the $K^{*}(1410)$ (which is generally not expected) or just too small statistics. However, quenched results using bilinear quark sources have been compatible with the $K^{*}(1410)$ [43].

We are planning to investigate this channel more thoroughly using a larger basis in the variational method and a larger set of ensembles in the future. We do not observe the $P$ wave scattering state $\pi K$ in our simulation.

\section{The $1^{+}$channel: $K_{1}$}

Similar to the strange $1^{-}$channel, we seem to miss the ground state $K_{1}(1270)$ in the strange $1^{+}$channel (see Fig. 21). Our result is in good agreement with the experimental first excitation $K_{1}(1400)$. In this channel the situation is clearer, due to missing isospin symmetry, one expects a mixing from the $1^{++}$and $1^{+-}$interpolators. In contrast to the latter case, these are both non-exotic. So far, we cannot say how strong the mixing is in our simulations, however, it was found that the mixing is weak down to pion masses of $400 \mathrm{MeV}$ [30]. Again, a more thorough investigation is hoped to shed some light on this issue. The results of ensemble $\mathrm{C}$ suggest that we do 


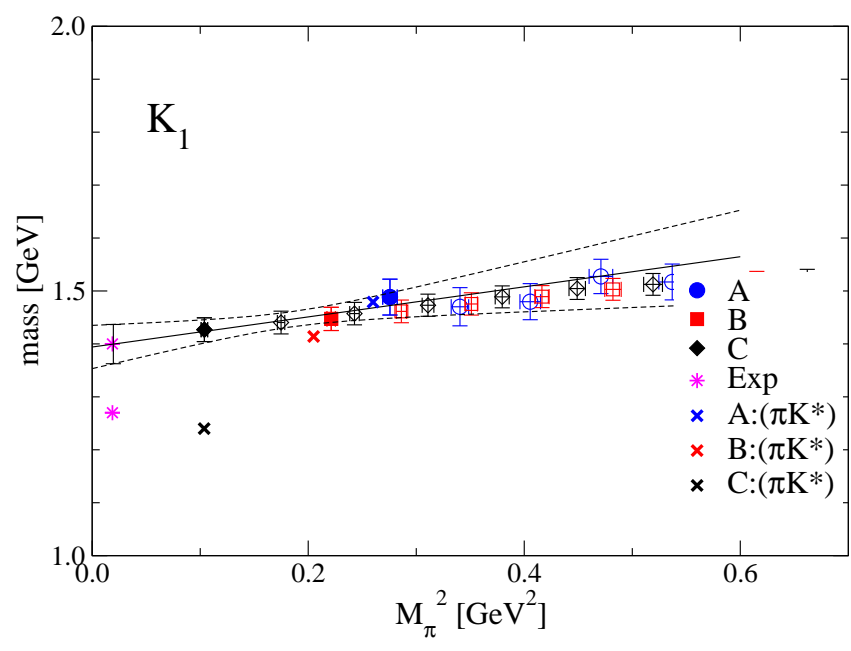

FIG. 21: Mass plot for the $1^{+}$channel $\left(K_{1}\right)$. Measured using interpolators $(13,14,15)$. The energy level of the $S$ wave scattering state $\pi K^{*}$ is close to the ground states of A and B, but much lower than the ground state of $\mathrm{C}$.

not observe the $S$ wave scattering state $\pi K^{*}$.

\section{The $2^{-}$channel: $K_{2}$}

As already discussed, there are two different orthogonal lattice irreducible representations for spin 2 channels. Again, we analyze the irreps independently and discuss the results. Similar to the corresponding light meson channels, we encounter large error bars in the strange $2^{-}$channel (see Fig. 22). The results are compatible with both the experimental $K_{2}(1770)$ and the $K_{2}(1820)$ in both representations, albeit with an error of slightly more than one $\sigma$ in case of $\mathrm{T}_{2}$. The $K_{2}(1580)$ is not completely confirmed experimentally so far. The lowest possible scattering state $\left(\pi K^{*}\right.$ in $P$ wave) is not observed in our simulation. Like in the spin 1 channels, we hope that enlarging the basis will improve the signal.

\section{The $2^{+}$channel: $K_{2}^{*}$}

In the $2^{+}$channel the signal is somewhat better than in the $2^{-}$channel. Here we find the result of irrep $\mathrm{T}_{2}$ closer to the experimental $K_{2}^{*}(1430)$ than the one of irrep E (see Figs. 23 and 24). However, the negative slope of the chiral extrapolation in irrep $\mathrm{E}$ is clearly an artifact of too small statistics (remember that only the three dynamical points enter the linear fit). A more complete basis and additional ensembles are expected to improve the calculation in this channel.
7. The $1^{--}$channel: $\phi$

The decay channels of $\phi$ suggest that it is an almost pure $s \bar{s}$-state. Therefore, in the partially quenched analysis we can read off our results for $\phi$ from the partially quenched data in the $1^{--}(\rho)$ channel without any chiral extrapolation (see Fig. 17). As already discussed, the ground state reproduces $\phi(1020)$ nicely, which can be seen as an affirmative cross-check for our method to set the strange quark mass parameter. Reading off the excited state we find some deviation from the experimental value, explicitly shown in Fig. 29 in the summary. The origin of this discrepancy may be due to neglected disconnected diagrams or just lie in the weakness of the corresponding effective mass plateau. Difficulties with excitations are found also in related channels. E.g., in the $1^{-}$channel we miss the first excitation of $K^{*}$ and in the light $1^{--}$channel the excitation of $\rho$ is quite noisy.

\section{Baryons with strange quarks}

$$
\text { 1. } \Sigma \text { positive parity }
$$

The interpolators of $\Sigma$ and $\Xi$ have the same Dirac structure as the nucleon interpolator, they just differ by the flavor content. Hence we use similar sets of interpolators in the variational method. We obtain a ground state and two excited states in the $\Sigma$ positive parity channel (see Fig. 25). The ground state result is in satisfactory agreement with experimental data. This is another affirmative cross-check of the setting of the strange quark mass parameter. The excitations are too high, which may be due to finite volume effects, as we have discussed already. Analogously to previous channels, we do not believe to see contributions from the $P$ wave scattering state $K N$.

\section{2. $\Sigma$ negative parity}

We find a ground state and two excitations in the $\Sigma$ negative parity channel (see Fig. 26). Similar to the nucleon negative parity channel, the first excitation is very close to the ground state, which is in qualitative agreement with the experimental $\Sigma(1620)$ and $\Sigma(1750)$. The ground state mass agrees with experiment within error bars, though being a bit low, as in all other our negative parity baryon channels. The second excitation could be compatible with the observed $\Sigma(2000)$, after correction of finite volume effects. $\Sigma(1620), \Sigma(1750)$ and $\Sigma(2000)$ are classified with 2, 3 and 1 stars, respectively, by the Particle Data Group [1]. We can confirm the two lower ones qualitatively and the existence of an excitation in the vicinity of $2000 \mathrm{MeV}$.

In nature, the $S$ wave scattering state $K N$, which is lighter than the 1-particle ground state, appears in the $\Sigma$ negative parity channel. We analyze the eigenvec- 

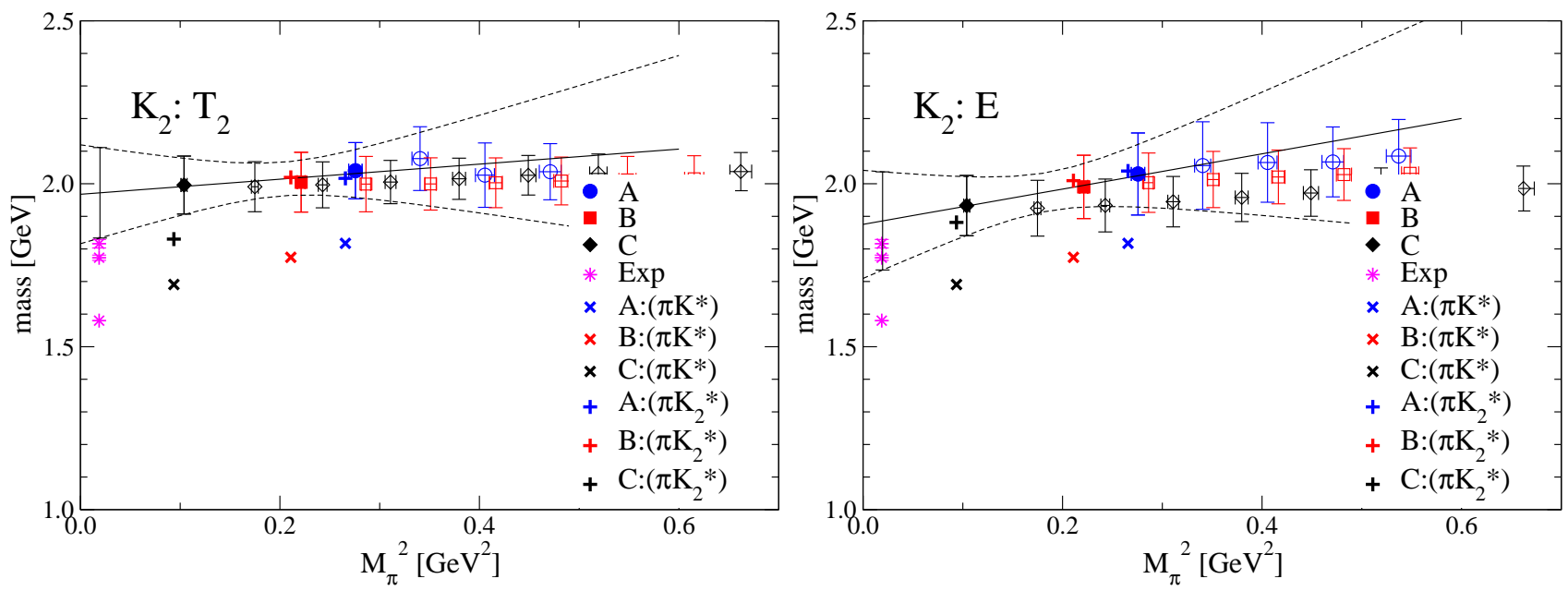

FIG. 22: Mass plot for the $2^{-}$channel $\left(K_{2}\right)$, ground state. Lhs: Irrep $\mathrm{T}_{2}$, measured using interpolators $(1,3)$. Rhs: Irrep E, measured using interpolators $(1,3)$. The experimental $K_{2}(1580)$ is not completely confirmed so far. The energy levels of the scattering states $\pi K_{2}^{*}$ ( $S$ wave, dominant in the experiment) and $\pi K^{*}$ ( $P$ wave) are shown.

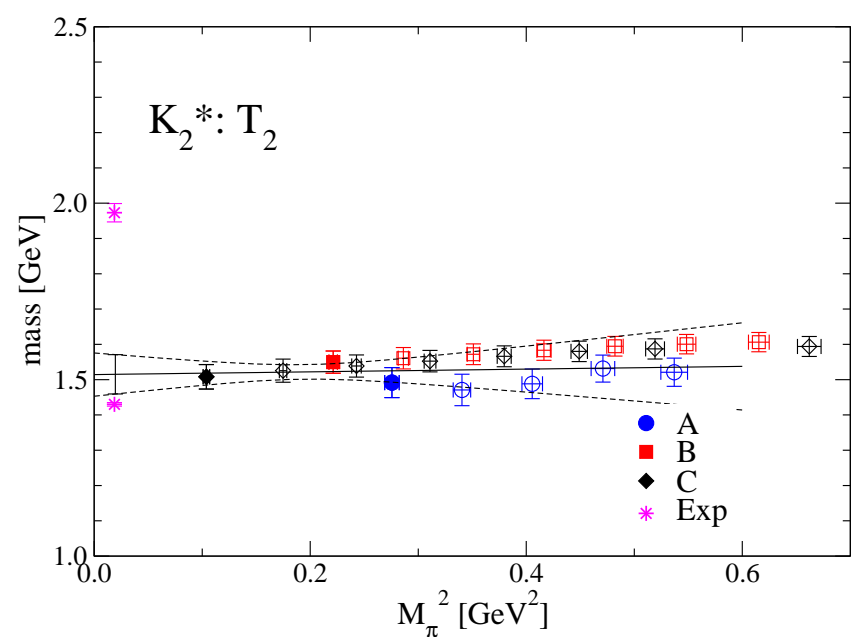

FIG. 23: Mass plot for the $2^{+}$channel $\left(K_{2}^{*}\right)$ in irrep $\mathrm{T}_{2}$, ground state. Measured using interpolators $(1,7)$ in $\mathrm{A},(3,5)$ in $\mathrm{B},(2,4,5,6)$ in $\mathrm{C}$.

tors analogously to the nucleon negative parity channel. Again, we find that in all three ensembles the ground state is dominated by the second Dirac structure $\left(\chi_{2}\right)$, while the first excitation is an almost pure $\chi_{1}$ state (see Table $\mathrm{X}$. The partially quenched points show the same behavior. One may conclude that no level crossing of the lowest two states is observed for pion masses in the range of 320 to $520 \mathrm{MeV}$. As in the nucleon negative parity channel, we find that the low results for masses at small pion masses may be explained by the presence of a scattering state, but the eigenvectors do not confirm this picture. Since the argumentation based on the eigenvectors seems to be more reliable, we believe to see an almost pure 1-particle state and quote the corresponding chiral extrapolation in the summary.

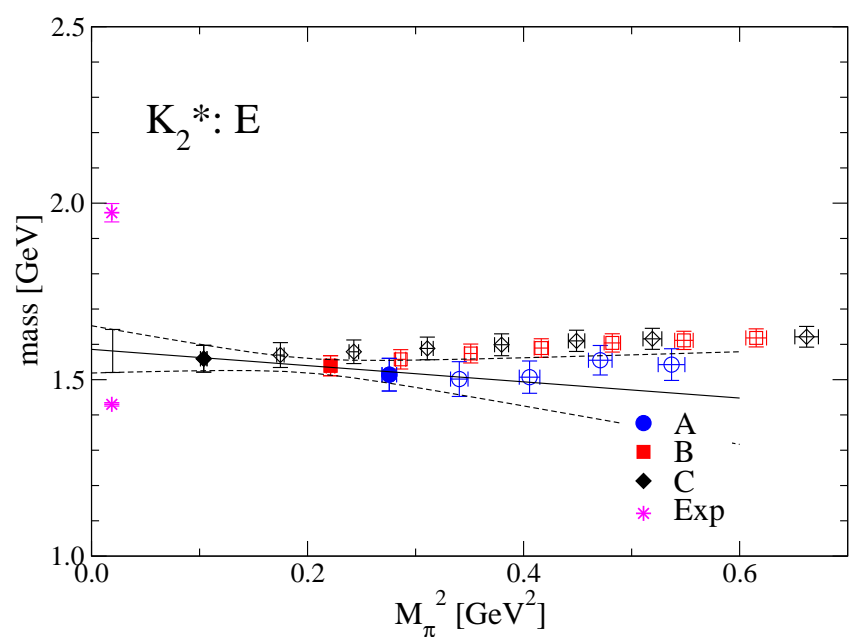

FIG. 24: Mass plot for the $2^{+}$channel $\left(K_{2}^{*}\right)$ in irrep E, ground state. Measured using interpolators $(2,3,9)$ in $\mathrm{A}$ and $\mathrm{C}$ and $(3,4,9)$ in $\mathrm{B}$.

\section{3. $\Xi$ positive parity}

In the $\Xi$ positive parity channel we obtain a ground state with rather small error which is in very good agreement with the experimental $\Xi$ ground state (see Fig. 27). We also get a prediction for a first excited state with comparatively small error bar in the range of 2200 to 2400 $\mathrm{MeV}$, which after correction of finite volume effects could be compatible with the $\Xi(2120)$ (1 stars) or the $\Xi(2250)$ (2 stars), where both states are listed by the particle data group stating neither spin nor parity. Analogous to previous cases, we do not believe to see measurable contributions from the $P$ wave scattering state $K \Sigma$. 


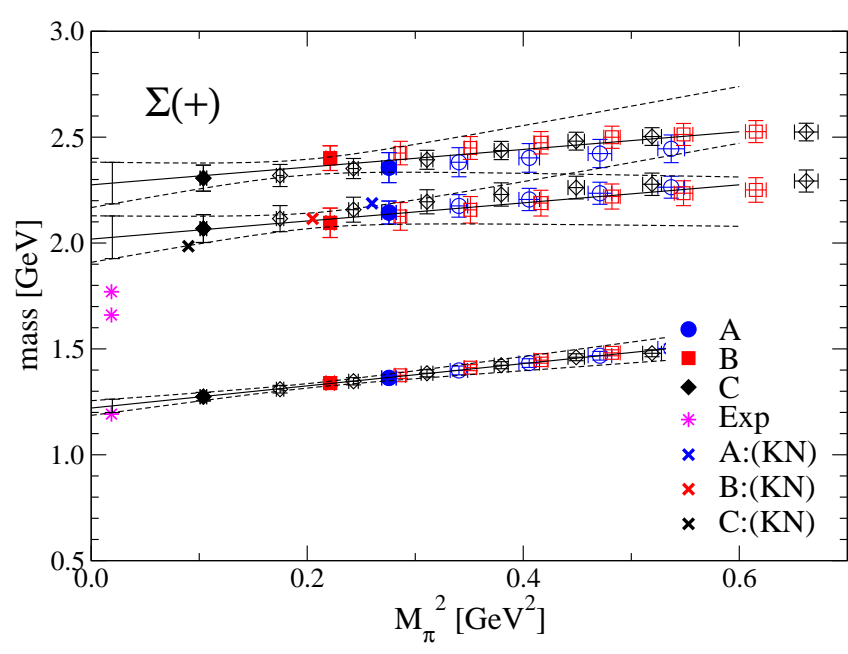

FIG. 25: Mass plot for the $\Sigma$ positive parity channel, ground state and two excitations. Ground state measured using interpolators $(1,6,17,20)$, first excitation measured using $(1,6,20)$, second excitation measured using $(3,4,8,9,13)$. The energy level of the $P$ wave scattering state $N K$ is very close to the first excitation in all three ensembles. For better identification, we display the scattering states slightly shifted to the left.

\section{4. $\Xi$ negative parity}

In the negative parity channel there is not even one established state listed by the Particle Data Group. We observe a ground state and a close first excitation in the range of 1550 to $1800 \mathrm{MeV}$, both with relatively small error (similar to the quenched results [44]). These states may match the $\Xi(1620)$ (1 stars) and the $\Xi(1690)$ (3 stars). There is a signal of a second excitation around $2400 \mathrm{MeV}$ as well, albeit with rather large statistical uncertainty and suffering from finite volume effects. We found it crucial to include all three baryon Dirac structures in the variational method in order to obtain both excitations.

Compared to quenched results of [44], we extracted an additional excited state in the present work.

\section{5. $\Omega$ positive parity}

As already discussed, we use our result for $\Omega(1672)$ to set the strange quark mass parameter (see Fig. [16). Similar to the case of $\phi$, we read off results for $\Omega$ from partially quenched data for $\Delta$, without any chiral extrapolation. In addition to $\Omega(1672)$, we obtain a first excitation in the positive parity channel (explicitely shown in Fig. 29 in the summary). Since there is no extrapolation involved, its statistical error is rather small, however, the true mass value may be somewhat smaller due to finite volume effects. The Particle Data Group lists $\Omega(2250)$ (3 stars), $\Omega(2380)$ (2 stars) and $\Omega(2470)$ (2 stars), stating neither spin nor parity. Taking into account finite vol-

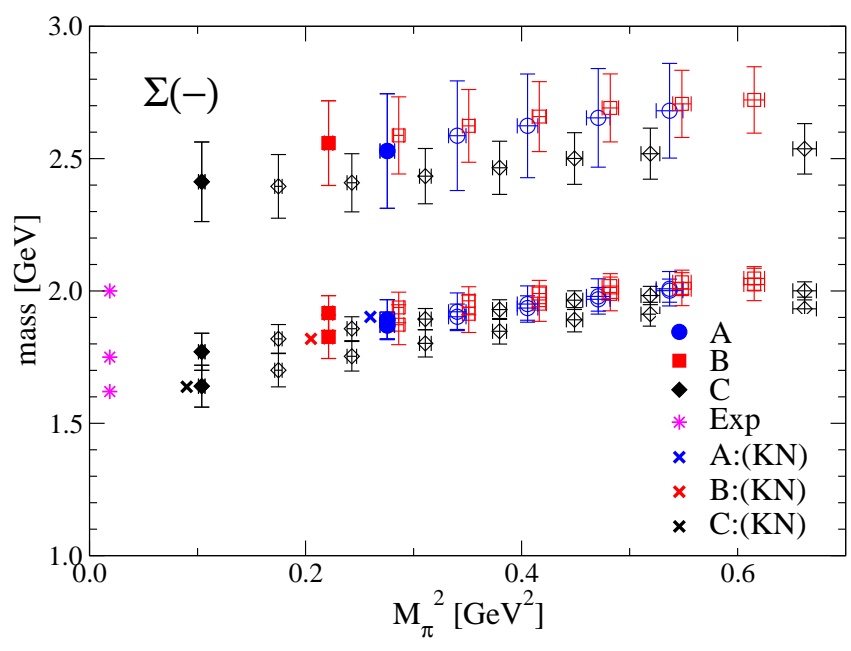

FIG. 26: Mass plot for the $\Sigma$ negative parity channel. Measured using interpolators $(1,9,10,12)$. The energy level of the $S$ wave scattering state $K N$ is very close to the ground state in all three ensembles (also to the first excitation in case of ensemble A). For better identification, we display the scattering states slightly shifted to the left. The results would be compatible with an interpretation in terms of a level crossing of the 1- and 2-particle states. However, analogously to the nucleon negative parity channel, the eigenvectors contradict this picture. For clarity, the chiral extrapolation is omitted in the figure.

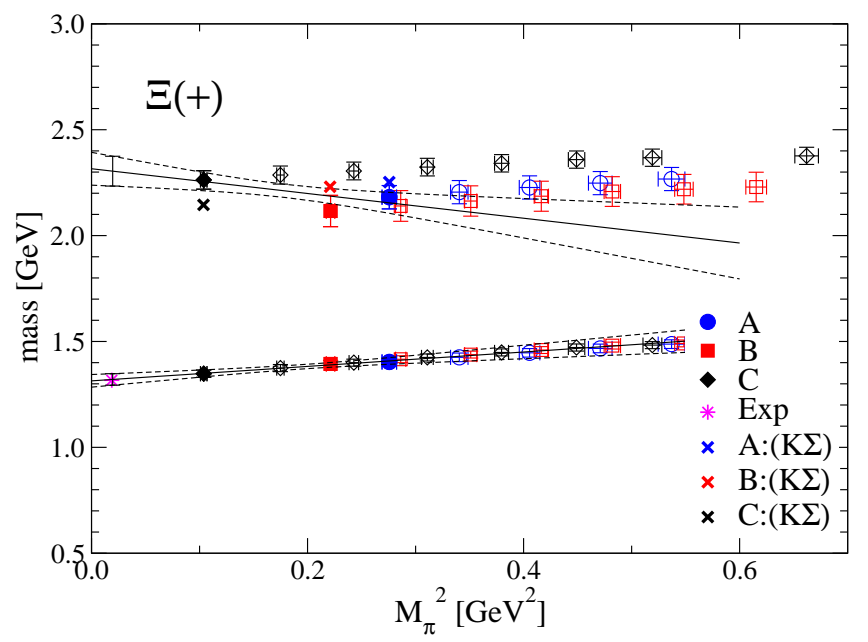

FIG. 27: Mass plot for the $\Xi$ positive parity channel. Measured using interpolators $(3,4,9,13,16)$. The energy level of the $P$ wave scattering state $K \Sigma$ is very close to the first excitation in all three ensembles.

ume effects, the result for the excitation may turn out to be compatible with $\Omega(2250)$.

\section{6. $\Omega$ negative parity}

The Particle Data Group does not list any established state in the $\Omega$ negative parity channel. We observe a 


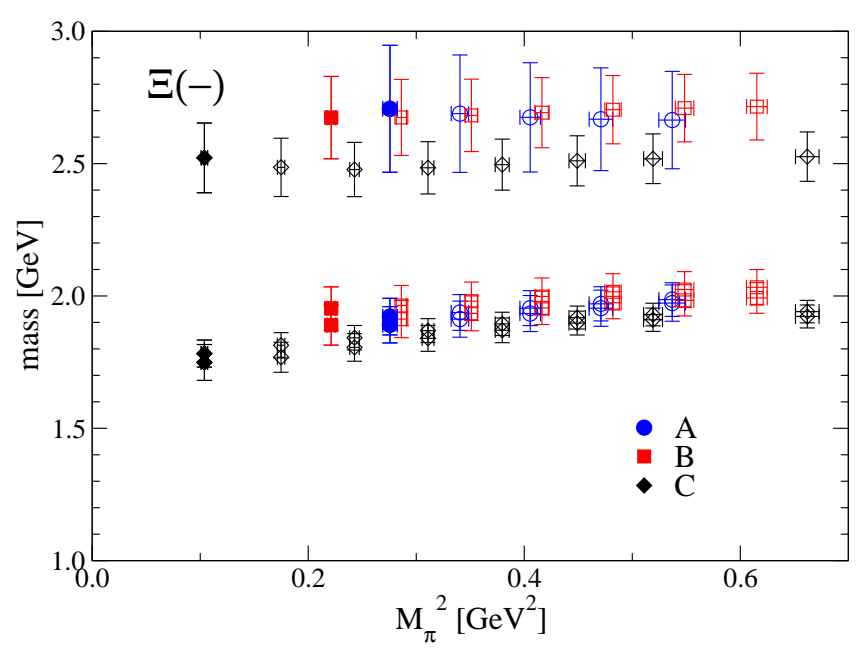

FIG. 28: Mass plot for the $\Xi$ negative parity channel. Measured using interpolators $(4,9,10,14,23)$ in $\mathrm{A}$ and $\mathrm{B}$, $(3,7,9,10,14)$ in C. For clarity, the chiral extrapolation is omitted in the figure.

ground state in the range of 2050 to $2100 \mathrm{MeV}$ (compare Fig. 15. explicitely shown in Fig. 29 in the summary). Such a state has also been observed in the quenched study [44]. It does not fit to any state listed by the Particle Data Group.

\section{CONCLUSION}

We have presented results of hadron spectroscopy using the Chirally Improved Dirac operator on lattices of size $16^{3} \times 32$ with two mass-degenerate light sea quarks. Three ensembles with pion masses of 322(5), 470(4) and $525(7) \mathrm{MeV}$ and lattice spacings all close to $0.15 \mathrm{fm}$ have been investigated. This allows for a naive chiral extrapolation in the mass-dependent scheme, but neither for a continuum nor a thermodynamic limit. We have discussed possible systematic effects in Sect. IV] Systematic uncertainty due to discretization effects is not explored explicitely and may be non-negligible for some observables. Finite volume effects are discussed only qualitatively, where we find some indications in case of excited baryons.

We have shown results for ground states and excited states for several meson and baryon channels, including spin 2 mesons constructed by the use of derivative sources. The spectrum of strange hadrons was accessed by using partially quenched strange quarks. Possible effects from partial quenching have been discussed briefly and they seem to play no important role for most of our final results. However, including strange sea quarks in the simulation would be desirable in order to reduce possible sources of systematic errors. The value of the strange quark mass parameter was set by identification of the partially quenched positive parity $\Delta$ with the $\Omega(1672)$.

The results are summarized in Fig. 29. Several of the

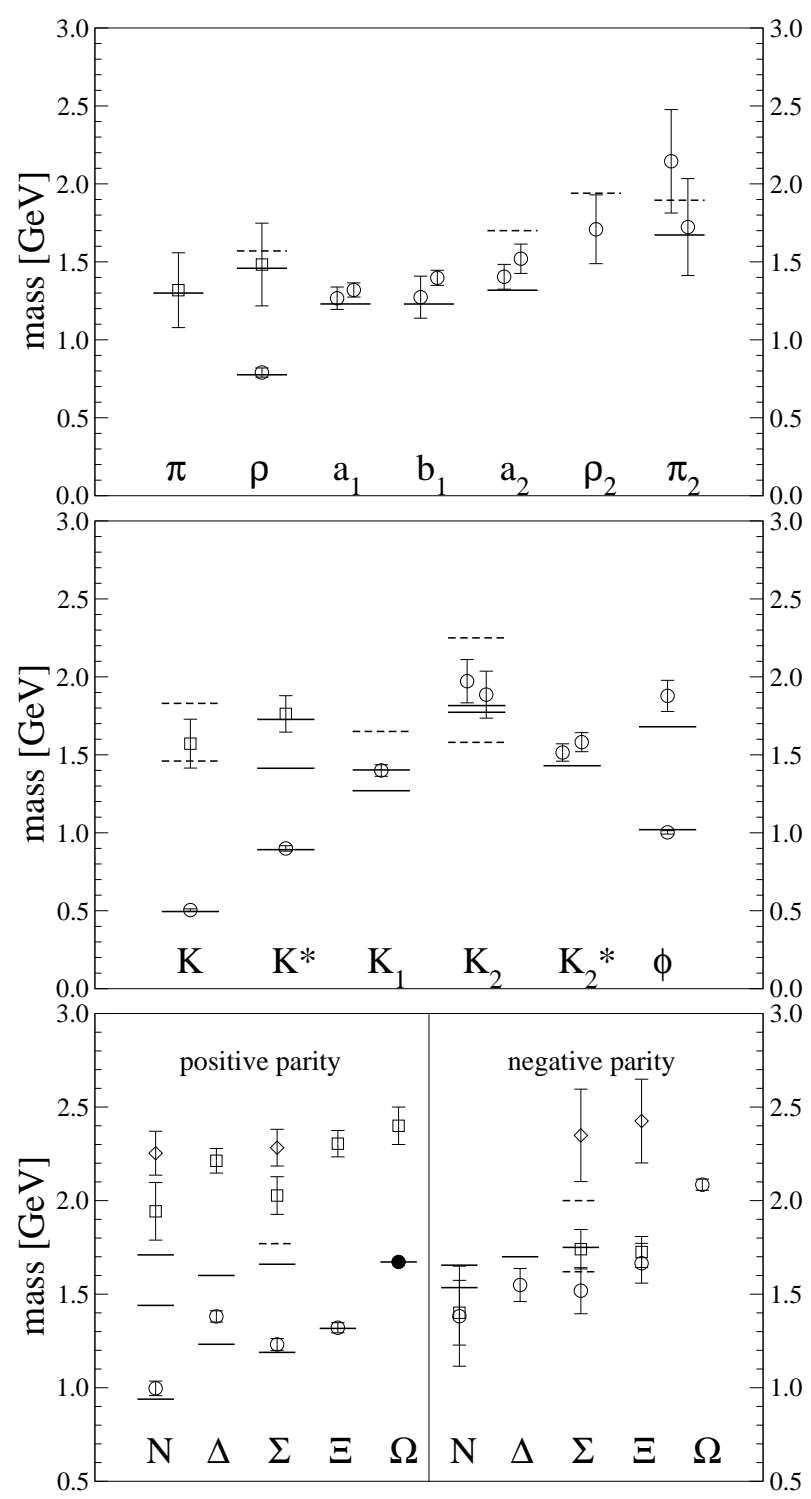

FIG. 29: Collection of the results for light meson masses (top), strange meson masses (middle) and baryon masses (bottom). Indicated errors are of purely statistical nature. Results are obtained by chiral extrapolation of dynamical data linear in the pion mass squared (except for $\phi$ and $\Omega$ hadrons). Experimental values listed by the Particle Data Group [1] are denoted by horizontal lines, the ones needing confirmation by dashed lines. Circles indicate ground states, squares first excitations and diamonds second excitations. Results shown aside each other are obtained using different sets of interpolators aiming for the same state. In the spin 2 channels, results from $\mathrm{T}_{2}$ interpolators are shown on the left, results from $\mathrm{E}$ on the right. In the $\rho_{2}$ channel we have results only from E interpolators. Strange quarks are implemented by partial quenching, the strange quark mass parameter is set by identification of a partially quenched $\Delta$ with $\Omega(1670)$. Excited baryons which come out too high seem to systematically suffer from finite volume effects. Higher statistics will be needed to establish if the results for the ground states in the negative parity baryon channels are compatible with experiment or whether the results are systematically too low. Results for scalar mesons are omitted in this figure, since the particle content of the corresponding observed states is too uncertain. 
experimentally known ground states are reproduced with fairly high precision $\left(\rho, K^{0}, K^{*}, N\right.$ and $\Sigma$ positive parity). In addition, various radial excitations are found $\left(\pi, \rho, K^{*}, \Delta\right.$ positive parity, $N, \Sigma$ and $\Xi$ both parities). We discussed the possible appearance of scattering states in various channels. The coupling of our interpolators to many-particle states seems to be weak and such states are barely, if at all, identifiable. Only in the light scalar channel the partially quenched data suggest a large contribution from an $S$ channel 2-particle state of pseudoscalars. However, at the dynamical point no clear statement is possible. In the negative parity nucleon and $\Sigma$ channels, the eigenvectors do not confirm the picture of the $S$ wave 2-particle states, either, although such an admixture cannot be completely excluded. A clear interpretation of the particle content of the observed states is still missing.

Where possible, we discussed the influence of sea quarks by comparison to quenched results obtained from the same action. In particular the spectroscopy in the light scalar channel seems to benefit from dynamical quarks, nevertheless it remains a difficult channel. Also the results in the light vector channel are in slightly better agreement with experiment when sea quarks are included. However, in most channels we did not observe a significant difference between quenched and dynamical simulations. We stress that in most cases comparison is difficult since some other details of the simulations differ as well. Also we have to emphasize that in many observables the overall effect of the sea quarks may approximately cancel (e.g., by including their effect on the scale setting). In other words, different dynamics may partly show similar phenomenology. A similar mechanism possibly works in some of the strange hadrons, where our results are in good agreement with experiment, despite the partial quenching approximation of the strange quark.

Several channels are expected to benefit from enlarged statistics, especially radial and spin excitations, the pseudovector mesons and negative parity baryon channels. In the nucleon positive parity channel we observe excitations which are definitely higher than the expected Roper resonance. This problem may be due to finite size effects or the lack of explicit 2-particle interpolators in the analysis. Our data confirm the existence of some states of unclear status and predict some states which are not listed by the Particle Data Group so far, albeit with large statistical errors and also non-negligible systematic errors in some cases.

\section{Acknowledgments}

We would like to thank Christof Gattringer, Leonid Y. Glozman and Sasa Prelovsek for valuable discussions. The calculations have been performed on the SGI Altix 4700 of the Leibniz-Rechenzentrum Munich and on local clusters at ZID at the University of Graz. We thank these institutions for providing support. M.L. and
D.M. have been supported by "Fonds zur Förderung der Wissenschaftlichen Forschung in Österreich" (DK W1203-N08). D.M. acknowledges support by COSYFFE Projekt 41821486 (COSY-105) and by Natural Sciences and Engineering Research Council of Canada (NSERC) and G.P.E. and A.S. acknowledge support by the DFG project SFB/TR-55. 


\section{Appendix A: Tables of interpolators}

\section{Meson interpolators}

We list the used interpolators for each meson channel in Tables [I- IX] In case of the light mesons, symmetrization is performed for definite $C$-parity. Our strange meson correlation calculation lacks cross correlation matrix elements according to interpolators with different $C$ parity quantum numbers in the limit of degenerate quark masses. Therefore, when analyzing strange mesons, we have to restrict ourselves to subsets of interpolators sharing the same $J^{P C}$ quantum numbers in the limit of degenerate quark masses.

The letters $a$ and $b$ denote light or strange quarks; $n(w)$ denotes a Gaussian shaped narrow (wide) source and $\partial_{i}$ denotes a derivative source in spatial direction $i$ (see Sec. III). $\gamma_{t}$ is the gamma matrix in time direction, $\epsilon_{i j k}$ is the Levi-Civita symbol, $Q_{i j k}$ are Clebsch-Gordon coefficients, where all elements are zero except $Q_{111}=$ $\frac{1}{\sqrt{2}}, Q_{122}=-\frac{1}{\sqrt{2}}, Q_{211}=-\frac{1}{\sqrt{6}}, Q_{222}=-\frac{1}{\sqrt{6}}$ and $Q_{233}=$ $\frac{2}{\sqrt{6}}$.

\begin{tabular}{c|c|l}
\hline \hline Interpolator & $0^{-}\left(K_{0}\right)$ & $0^{-+}(\pi)$ Sym. \\
\hline $\bar{a}_{n} \gamma_{5} b_{n}$ & $1_{0^{-}}$ & $1_{0^{-+}}=1_{0^{-}}$ \\
$\bar{a}_{n} \gamma_{5} b_{w}$ & $2_{0^{-}}$ & $2_{0^{-+}}=2_{0^{-}}+3_{0^{-}}$ \\
$\bar{a}_{w} \gamma_{5} b_{n}$ & $3_{0^{-}}$ & \\
$\bar{a}_{w} \gamma_{5} b_{w}$ & $4_{0^{-}}$ & $3_{0^{-+}}=4_{0^{-}}$ \\
$\bar{a}_{n} \gamma_{t} \gamma_{5} b_{n}$ & $5_{0^{-}}$ & $4_{0^{-+}}=5_{0^{-}}$ \\
$\bar{a}_{n} \gamma_{t} \gamma_{5} b_{w}$ & $6_{0^{-}}$ & $5_{0^{-+}}=6_{0^{-}}+7_{0^{-}}$ \\
$\bar{a}_{w} \gamma_{t} \gamma_{5} b_{n}$ & $7_{0^{-}}$ & \\
$\bar{a}_{w} \gamma_{t} \gamma_{5} b_{w}$ & $8_{0^{-}}$ & $6_{0^{-+}}=8_{0^{-}}$ \\
$\bar{a}_{\partial_{i}} \gamma_{i} \gamma_{5} b_{n}$ & $9_{0^{-}}$ & $7_{0^{-+}}=9_{0^{-}}+11_{0^{-}}$ \\
$\bar{a}_{\partial_{i}} \gamma_{i} \gamma_{5} b_{w}$ & $10_{0^{-}}$ & $8_{0^{-+}}=10_{0^{-}}+12_{0^{-}}$ \\
$\bar{a}_{n} \gamma_{i} \gamma_{5} b_{\partial_{i}}$ & $11_{0^{-}}$ & \\
$\bar{a}_{w} \gamma_{i} \gamma_{5} b_{\partial_{i}}$ & $12_{0^{-}}$ & \\
$\bar{a}_{\partial_{i}} \gamma_{i} \gamma_{t} \gamma_{5} b_{n}$ & $13_{0^{-}}$ & $9_{0^{-+}}=13_{0^{-}}-15_{0^{-}}$ \\
$\bar{a}_{\partial_{i}} \gamma_{i} \gamma_{t} \gamma_{5} b_{w}$ & $14_{0^{-}}$ & $10_{0^{-+}}=14_{0^{-}}-16_{0^{-}}$ \\
$\bar{a}_{n} \gamma_{i} \gamma_{t} \gamma_{5} b_{\partial_{i}}$ & $15_{0^{-}}$ & \\
$\bar{a}_{w} \gamma_{i} \gamma_{t} \gamma_{5} b_{\partial_{i}}$ & $16_{0^{-}}$ & \\
$\bar{a}_{\partial_{i}} \gamma_{5} b_{\partial_{i}}$ & $17_{0^{-}}$ & $11_{0^{-+}}=17_{0^{-}}$ \\
$\bar{a}_{\partial_{i}} \gamma_{t} \gamma_{5} b_{\partial_{i}}$ & $18_{0^{-}}$ & $12_{0^{-+}}=18_{0^{-}}$ \\
\hline \hline
\end{tabular}

TABLE II: Pseudoscalar interpolators from irrep $A_{1}$. The unsymmetrized interpolator, the corresponding number in the strange meson channel $0^{-}$, the number and the symmetrization in terms of the $0^{-}$interpolators are given for the light meson channel $0^{-+}$.

\begin{tabular}{c|c|l}
\hline \hline Interpolator & $0^{+}\left(K_{0}^{*}\right)$ & $0^{++}\left(a_{0}\right)$ Sym. \\
\hline $\bar{a}_{n} b_{n}$ & $1_{0^{+}}$ & $1_{0^{++}}=1_{0^{+}}$ \\
$\bar{a}_{n} b_{w}$ & $2_{0^{+}}$ & $2_{0^{++}}=2_{0^{+}}+3_{0^{+}}$ \\
$\bar{a}_{w} b_{n}$ & $3_{0^{+}}$ & \\
$\bar{a}_{w} b_{w}$ & $4_{0^{+}}$ & $3_{0^{++}}=4_{0^{+}}$ \\
$\bar{a}_{\partial_{i}} \gamma_{i} b_{n}$ & $5_{0^{+}}$ & $4_{0^{++}}=5_{0^{+}}-7_{0^{+}}$ \\
$\bar{a}_{\partial_{i}} \gamma_{i} b_{w}$ & $6_{0^{+}}$ & $5_{0^{++}}=6_{0^{+}}-8_{0^{+}}$ \\
$\bar{a}_{n} \gamma_{i} b_{\partial_{i}}$ & $7_{0^{+}}$ & \\
$\bar{a}_{w} \gamma_{i} b_{\partial_{i}}$ & $8_{0^{+}}$ & \\
$\bar{a}_{\partial_{i}} \gamma_{i} \gamma_{t} b_{n}$ & $9_{0^{+}}$ & $6_{0^{++}}=9_{0^{+}}-11_{0^{+}}$ \\
$\bar{a}_{\partial_{i}} \gamma_{i} \gamma_{t} b_{w}$ & $10_{0^{+}}$ & $7_{0^{++}}=10_{0^{+}}-12_{0^{+}}$ \\
$\bar{a}_{n} \gamma_{i} \gamma_{t} b_{\partial_{i}}$ & $11_{0^{+}}$ & \\
$\bar{a}_{w} \gamma_{i} \gamma_{t} b_{\partial_{i}}$ & $12_{0^{+}}$ & \\
$\bar{a}_{\partial_{i}} b_{\partial_{i}}$ & $13_{0^{+}}$ & $8_{0^{++}}=13_{0^{+}}$ \\
\hline \hline
\end{tabular}

TABLE III: Scalar interpolators from irrep $A_{1}$. The unsymmetrized interpolator, the corresponding number in the strange meson channel $0^{+}$, the number and the symmetrization in terms of the $0^{-}$interpolators are given for the light meson channel $0^{++}$.

\begin{tabular}{|c|c|c|c|}
\hline Interpolator & $1^{-}\left(K^{*}\right)$ & $1^{--}$ & $(\rho)$ Sym. \\
\hline $\bar{a}_{n} \gamma_{k} b_{n}$ & $1_{1^{-}}$ & $1_{1^{--}}$ & $=1_{1^{-}}$ \\
\hline $\bar{a}_{n} \gamma_{k} b_{w}$ & $2_{1-}$ & $21^{--}$ & $=2_{1^{-}}+3_{1-}$ \\
\hline $\bar{a}_{w} \gamma_{k} b_{n}$ & $3_{1-}$ & & \\
\hline $\bar{a}_{w} \gamma_{k} b_{w}$ & $4_{1-}$ & $31^{--}$ & $=4_{1^{-}}$ \\
\hline $\bar{a}_{n} \gamma_{k} \gamma_{t} b_{n}$ & $5_{1-}$ & $4_{1--}$ & $=5_{1^{-}}$ \\
\hline $\bar{a}_{n} \gamma_{k} \gamma_{t} b_{w}$ & $6_{1^{-}}$ & $5_{1--}$ & $=6_{1^{-}}+7_{1^{-}}$ \\
\hline $\bar{a}_{w} \gamma_{k} \gamma_{t} b_{n}$ & $7_{1-}$ & & \\
\hline $\bar{a}_{w} \gamma_{k} \gamma_{t} b_{w}$ & $8_{1-}$ & $61^{--}$ & $=8_{1^{-}}$ \\
\hline $\bar{a}_{\partial_{k}} b_{n}$ & $9_{1-}$ & $7_{1--}$ & $=9_{1^{-}}-11_{1^{-}}$ \\
\hline $\bar{a}_{\partial_{k}} b_{w}$ & $10_{1^{-}}$ & $81^{--}$ & $=10_{1^{-}}-12_{1^{-}}$ \\
\hline $\bar{a}_{n} b_{\partial_{k}}$ & $11_{1^{-}}$ & & \\
\hline $\bar{a}_{w} b_{\partial_{k}}$ & $12_{1-}$ & & \\
\hline $\bar{a}_{\partial_{k}} \gamma_{t} b_{n}$ & $13_{1-}$ & $91^{--}$ & $=13_{1-}+15_{1-}$ \\
\hline $\bar{a}_{\partial_{k}} \gamma_{t} b_{w}$ & $14_{1-}$ & $10_{1--}$ & $=14_{1^{-}}+16_{1-}$ \\
\hline $\bar{a}_{n} \gamma_{t} b_{\partial_{k}}$ & $15_{1-}$ & & \\
\hline $\bar{a}_{w} \gamma_{t} b_{\partial_{k}}$ & $16_{1-}$ & & \\
\hline $\bar{a}_{\partial_{i}} \gamma_{k} b_{\partial_{i}}$ & $17_{1^{-}}$ & $11_{1^{--}}$ & $=17_{1^{-}}$ \\
\hline $\bar{a}_{\partial_{i}} \gamma_{k} \gamma_{t} b_{\partial_{i}}$ & $18_{1-}$ & $12_{1--}$ & $=18_{1^{-}}$ \\
\hline $\bar{a}_{\partial_{k}} \epsilon_{i j k} \gamma_{j} \gamma_{5} b_{n}$ & $19_{1-}$ & $13_{1^{--}}$ & $=19_{1^{-}}-21_{1^{-}}$ \\
\hline $\bar{a}_{\partial_{k}} \epsilon_{i j k} \gamma_{j} \gamma_{5} b_{w}$ & $20_{1-}$ & $14_{1--}$ & $=20_{1^{-}}-22_{1^{-}}$ \\
\hline $\bar{a}_{n} \epsilon_{i j k} \gamma_{j} \gamma_{5} b_{\partial_{k}}$ & $21_{1^{-}}$ & & \\
\hline $\bar{a}_{w} \epsilon_{i j k} \gamma_{j} \gamma_{5} b_{\partial_{k}}$ & $22_{1-}$ & & \\
\hline
\end{tabular}

TABLE IV: Vector interpolators from irrep $T_{1}$. The unsymmetrized interpolator, the corresponding number in the strange meson channel $1^{-}$, the number and the symmetrization in terms of the $1^{-}$interpolators are given for the light meson channel $1^{--}$. 


\begin{tabular}{|c|c|c|c|c|}
\hline Interpolator & $1^{+}\left(K_{1}\right)$ & $1^{++}$ & $\left(a_{1}\right)$ Sym. & $1^{+-}\left(b_{1}\right)$ Sym. \\
\hline $\bar{a}_{n} \gamma_{k} \gamma_{5} b_{n}$ & $1_{1+}$ & $1_{1++}$ & $=1_{1+}$ & \\
\hline $\bar{a}_{n} \gamma_{k} \gamma_{5} b_{w}$ & $21^{+}$ & $22_{1++}$ & $=2_{1+}+3_{1+}$ & \\
\hline $\bar{a}_{w} \gamma_{k} \gamma_{5} b_{n}$ & $3_{1+}$ & & & \\
\hline $\bar{a}_{w} \gamma_{k} \gamma_{5} b_{w}$ & $4_{1+}$ & $3_{1++}$ & $=4_{1+}$ & \\
\hline $\bar{a}_{\partial_{k}} \gamma_{5} b_{n}$ & $5_{1+}$ & $4_{1++}$ & $=5_{1+}+7_{1+}$ & $4_{1+-}=5_{1+}-7_{1+}$ \\
\hline $\bar{a}_{\partial_{k}} \gamma_{5} b_{w}$ & $6_{1+}$ & $5_{1++}$ & $=6_{1+}+8_{1+}$ & $5_{1+-}=6_{1+}-8_{1+}$ \\
\hline $\bar{a}_{n} \gamma_{5} b_{\partial_{k}}$ & $7_{1+}$ & & & \\
\hline $\bar{a}_{w} \gamma_{5} b_{\partial_{k}}$ & $8_{1+}+$ & & & \\
\hline $\bar{a}_{\partial_{k}} \gamma_{t} \gamma_{5} b_{n}$ & $9_{1+}$ & $66_{1++}$ & $=9_{1+}+11_{1+}$ & $6_{1+-}=9_{1+}-11_{1+}$ \\
\hline $\bar{a}_{\partial_{k}} \gamma_{t} \gamma_{5} b_{w}$ & $10_{1+}$ & $7_{1++}$ & $=10_{1+}+12_{1+}$ & $7_{1+-}=10_{1+}-12_{1+}$ \\
\hline $\bar{a}_{n} \gamma_{t} \gamma_{5} b_{\partial_{k}}$ & $11_{1+}$ & & & \\
\hline $\bar{a}_{w} \gamma_{t} \gamma_{5} b_{\partial_{k}}$ & $12_{1+}$ & & & \\
\hline $\bar{a}_{\partial_{i}} \gamma_{k} \gamma_{5} b_{\partial_{i}}$ & $13_{1+}$ & $88_{1++}$ & $=13_{1+}$ & \\
\hline$\epsilon_{i j k} \bar{a}_{\partial_{k}} \gamma_{j} b_{n}$ & $14_{1+}$ & $9_{1++}$ & $=14_{1+}-16_{1+}$ & \\
\hline$\epsilon_{i j k} \bar{a}_{\partial_{k}} \gamma_{j} b_{w}$ & $15_{1+}$ & $10_{1++}$ & $=15_{1+}-17_{1+}$ & \\
\hline$\epsilon_{i j k} \bar{a}_{n} \gamma_{j} b_{\partial_{k}}$ & $16_{1+}$ & & & \\
\hline$\epsilon_{i j k} \bar{a}_{w} \gamma_{j} b_{\partial_{k}}$ & $17_{1+}$ & & & \\
\hline$\epsilon_{i j k} \bar{a}_{\partial_{k}} \gamma_{j} \gamma_{t} b_{n}$ & $18_{1+}$ & $11_{1++}$ & $=18_{1+}-20_{1+}$ & \\
\hline$\epsilon_{i j k} \bar{a}_{\partial_{k}} \gamma_{j} \gamma_{t} b_{w}$ & $19_{1+}$ & $12_{1++}$ & $=19_{1+}-21_{1+}$ & \\
\hline$\epsilon_{i j k} \bar{a}_{n} \gamma_{j} \gamma_{t} b_{\partial_{k}}$ & $20_{1+}$ & & & \\
\hline$\epsilon_{i j k} \bar{a}_{w} \gamma_{j} \gamma_{t} b_{\partial_{k}}$ & $21_{1+}$ & & & \\
\hline $\bar{a}_{n} \gamma_{k} \gamma_{t} \gamma_{5} b_{n}$ & $22_{1+}$ & & & $1_{1+-}=22_{1+}$ \\
\hline $\bar{a}_{n} \gamma_{k} \gamma_{t} \gamma_{5} b_{w}$ & $23_{1+}$ & & & $2_{1+-}=23_{1+}+24_{1+}$ \\
\hline $\bar{a}_{w} \gamma_{k} \gamma_{t} \gamma_{5} b_{n}$ & $24_{1+}$ & & & \\
\hline $\bar{a}_{w} \gamma_{k} \gamma_{t} \gamma_{5} b_{w}$ & $25_{1+}$ & & & $3_{1+-}=25_{1+}$ \\
\hline $\bar{a}_{\partial_{i}} \gamma_{k} \gamma_{t} \gamma_{5} b_{\partial_{i}}$ & $26_{1+}$ & & & $8_{1+-}=26_{1+}$ \\
\hline
\end{tabular}

TABLE V: Pseudovector interpolators from irrep $T_{1}$. The unsymmetrized interpolator, the corresponding number in the strange meson channel $1^{+}$, the number and the symmetrization in terms of the $1^{+}$interpolators are given for the light meson channels $1^{++}$and $1^{+-}$.

\begin{tabular}{c|c|l|l}
\hline \hline Interpolator & $2_{T_{2}}^{-}\left(K_{2}\right)$ & $2_{T_{2}}^{--}\left(\rho_{2}\right)$ Sym. & $2_{T_{2}}^{-+}\left(\pi_{2}\right)$ Sym. \\
\hline$\left|\epsilon_{i j k}\right| \bar{a}_{\partial_{k}} \gamma_{j} \gamma_{5} b_{n}$ & $1_{2_{T_{2}}^{-}}$ & $1_{2_{T_{2}}^{-}}=1_{2_{T_{2}}^{-}}-3_{2_{T_{2}}}$ & \\
$\left|\epsilon_{i j k}\right| \bar{a}_{\partial_{k}} \gamma_{j} \gamma_{5} b_{w}$ & $2_{2_{T_{2}}^{-}}$ & $2_{2_{T_{2}}^{-}}=2_{2_{T_{2}}^{-}}-4_{2_{T_{2}}^{-}}$ & \\
$\left|\epsilon_{i j k}\right| \bar{a}_{n} \gamma_{j} \gamma_{5} b_{\partial_{k}}$ & $3_{2_{T_{2}}^{-}}$ & & \\
$\left|\epsilon_{i j k}\right| \bar{a}_{w} \gamma_{j} \gamma_{5} b_{\partial_{k}}$ & $4_{2_{T_{2}}^{-}}$ & & $1_{2_{T_{2}^{-}}^{-}}=5_{2_{T_{2}}^{-}-7_{2_{T_{2}}^{-}}}$ \\
$\left|\epsilon_{i j k}\right| \bar{a}_{\partial_{k}} \gamma_{j} \gamma_{t} \gamma_{5} b_{n}$ & $5_{2_{T_{2}}^{-}}$ & & $2_{T_{T_{2}}^{-}}=6_{2_{T_{2}}^{-}}-8_{2_{T_{2}}^{-}}$ \\
$\left|\epsilon_{i j k}\right| \bar{a}_{\partial_{k}} \gamma_{j} \gamma_{t} \gamma_{5} b_{w}$ & $6_{2_{T_{2}}^{-}}$ & & \\
$\left|\epsilon_{i j k}\right| \bar{a}_{n} \gamma_{j} \gamma_{t} \gamma_{5} b_{\partial_{k}}$ & $7_{2_{T_{2}}^{-}}$ & & \\
$\left|\epsilon_{i j k}\right| \bar{a}_{w} \gamma_{j} \gamma_{t} \gamma_{5} b_{\partial_{k}}$ & $8_{2_{T_{2}}^{-}}$ & & \\
\hline \hline
\end{tabular}

TABLE VI: Pseudotensor interpolators from irrep $T_{2}$. The unsymmetrized interpolator, the corresponding number in the strange meson channel $2_{T_{2}}^{-}$, the number and the symmetrization in terms of the $2_{T_{2}}^{-}$interpolators are given for the light meson channels $2_{T_{2}}^{--}$and $2_{T_{2}}^{-+}$. 


\begin{tabular}{c|c|l|l}
\hline \hline Interpolator & $2_{E}^{-}\left(K_{2}\right)$ & $2_{E}^{--}\left(\rho_{2}\right)$ Sym. & $2_{E}^{-+}\left(\pi_{2}\right)$ Sym. \\
\hline$Q_{i j k} \bar{a}_{\partial_{k}} \gamma_{j} \gamma_{5} b_{n}$ & $1_{2_{E}^{-}}$ & $1_{2_{E}^{-}}=1_{2_{E}^{-}}-3_{2_{E}^{-}}$ & \\
$Q_{i j k} \bar{a}_{\partial_{k}} \gamma_{j} \gamma_{t} \gamma_{5} b_{n}$ & $2_{2_{E}^{-}}$ & $2_{2_{E}^{-}}=2_{2_{E}^{-}}-4_{2_{E}^{-}}$ & \\
$Q_{i j k} \bar{a}_{n} \gamma_{j} \gamma_{5} b_{\partial_{k}}$ & $3_{2_{E}^{-}}$ & & \\
$Q_{i j k} \bar{a}_{w} \gamma_{j} \gamma_{5} b_{\partial_{k}}$ & $4_{2_{E}^{-}}$ & & \\
$Q_{i j k} \bar{a}_{\partial_{k}} \gamma_{j} \gamma_{t} \gamma_{5} b_{n}$ & $5_{2_{E}^{-}}$ & & $1_{2_{E}^{-+}}=5_{2_{E}^{-}}-7_{2_{E}^{-}}$ \\
$Q_{i j k} \bar{a}_{\partial_{k}} \gamma_{j} \gamma_{t} \gamma_{5} b_{w}$ & $6_{2_{E}^{-}}$ & & $2_{2_{E}^{-}}=6_{2_{E}^{-}}-8_{2_{E}^{-}}$ \\
$Q_{i j k} \bar{a}_{n} \gamma_{j} \gamma_{t} \gamma_{5} b_{\partial_{k}}$ & $7_{2_{E}^{-}}$ & & \\
$Q_{i j k} \bar{a}_{w} \gamma_{j} \gamma_{t} \gamma_{5} b_{\partial_{k}}$ & $8_{2_{E}^{-}}$ & & \\
$Q_{i j k} \bar{a}_{\partial_{j}} \gamma_{5} b_{\partial_{k}}$ & $9_{2_{E}^{-}}$ & & \\
$Q_{i j k} \bar{a}_{\partial_{j}} \gamma_{t} \gamma_{5} b_{\partial_{k}}$ & $10_{2_{E}^{-}}$ & & $3_{2_{E}^{-}}=9_{2_{E}^{-}}$ \\
\hline \hline
\end{tabular}

TABLE VII: Pseudotensor interpolators from irrep E. The unsymmetrized interpolator, the corresponding number in the strange meson channel $2_{E}^{-}$, the number and the symmetrization in terms of the $2_{E}^{-}$interpolators are given for the light meson channels $2_{E}^{--}$and $2_{E}^{-+}$.

\begin{tabular}{c|c|l}
\hline \hline Interpolator & $2_{T_{2}}^{+}\left(K_{2}^{*}\right)$ & $2_{T_{2}}^{++}\left(a_{2}\right)$ Sym. \\
\hline$\left|\epsilon_{i j k}\right| \bar{a}_{\partial_{k}} \gamma_{j} b_{n}$ & $1_{2_{T_{2}}^{+}}$ & $1_{2_{T_{2}}^{++}}=1_{2_{T_{2}}^{+}}-3_{2_{T_{2}}}$ \\
$\left|\epsilon_{i j k}\right| \bar{a}_{\partial_{k}} \gamma_{j} b_{w}$ & $2_{2_{T_{2}}^{+}}$ & $2_{2_{T_{2}}^{++}}=2_{2_{T_{2}}^{+}}-4_{2_{T_{2}}^{+}}$ \\
$\left|\epsilon_{i j k}\right| \bar{a}_{n} \gamma_{j} b_{\partial_{k}}$ & $3_{2_{T_{2}}^{+}}$ & \\
$\left|\epsilon_{i j k}\right| \bar{a}_{w} \gamma_{j} b_{\partial_{k}}$ & $4_{2_{T_{2}}^{+}}$ & \\
$\left|\epsilon_{i j k}\right| \bar{a}_{\partial_{k}} \gamma_{j} \gamma_{t} b_{n}$ & $5_{2_{T_{2}}^{+}}$ & $3_{2_{T_{2}+}^{++}}=5_{2_{T_{2}}^{+}}-7_{2_{T_{2}}^{+}}$ \\
$\left|\epsilon_{i j k}\right| \bar{a}_{\partial_{k}} \gamma_{j} \gamma_{t} b_{w}$ & $6_{2_{T_{2}}^{+}}$ & $4_{2_{T_{2}}^{++}}=6_{2_{T_{2}}^{+}}-8_{2_{T_{2}}^{+}}$ \\
$\left|\epsilon_{i j k}\right| \bar{a}_{n} \gamma_{j} \gamma_{t} b_{\partial_{k}}$ & $7_{2_{T_{2}}^{+}}$ \\
$\left|\epsilon_{i j k}\right| \bar{a}_{w} \gamma_{j} \gamma_{t} b_{\partial_{k}}$ & $8_{2_{T_{2}}^{+}}$ \\
\hline \hline
\end{tabular}

TABLE VIII: Tensor interpolators from irrep $T_{2}$. The unsymmetrized interpolator, the corresponding number in the strange meson channel $2_{T_{2}}^{+}$, the number and the symmetrization in terms of the $2_{T_{2}}^{+}$interpolators are given for the light meson channel $2_{T_{2}}^{++}$. 


\begin{tabular}{c|c|l}
\hline \hline Interpolator & $2_{E}^{+}\left(K_{2}^{*}\right)$ & $2_{E}^{++}\left(a_{2}\right)$ Sym \\
\hline$Q_{i j k} \bar{a}_{\partial_{k}} \gamma_{j} b_{n}$ & $1_{2_{E}^{+}}$ & $1_{2_{E}^{++}}=1_{2_{E}^{+}}-3_{2_{E}^{+}}$ \\
$Q_{i j k} \bar{a}_{\partial_{k}} \gamma_{j} b_{w}$ & $2_{2_{E}^{+}}$ & $2_{2_{E}^{++}}=2_{2_{E}^{+}}-4_{2_{E}^{+}}$ \\
$Q_{i j k} \bar{a}_{n} \gamma_{j} b_{\partial_{k}}$ & $3_{2_{E}^{+}}$ & \\
$Q_{i j k} \bar{a}_{w} \gamma_{j} b_{\partial_{k}}$ & $4_{2_{E}^{+}}$ & \\
$Q_{i j k} \bar{a}_{\partial_{k}} \gamma_{j} \gamma_{t} b_{n}$ & $5_{2_{E}^{+}}$ & $3_{2_{E}^{++}}=5_{2_{E}^{+}}-7_{2_{E}^{+}}$ \\
$Q_{i j k} \bar{a}_{\partial_{k}} \gamma_{j} \gamma_{t} b_{w}$ & $6_{2_{E}^{+}}$ & $4_{2_{E}^{++}}=6_{2_{E}^{+}}-8_{2_{E}^{+}}$ \\
$Q_{i j k} \bar{a}_{n} \gamma_{j} \gamma_{t} b_{\partial_{k}}$ & $7_{2_{E}^{+}}$ & \\
$Q_{i j k} \bar{a}_{w} \gamma_{j} \gamma_{t} b_{\partial_{k}}$ & $8_{2_{E}^{+}}$ & \\
$Q_{i j k} \bar{a}_{\partial_{j}} b_{\partial_{k}}$ & $9_{2_{E}^{+}}$ & $5_{2_{E}^{++}}=9_{2_{E}^{+}}$ \\
\hline \hline
\end{tabular}

TABLE IX: Tensor interpolators from irrep $E$. The unsymmetrized interpolator, the corresponding number in the strange meson channel $2_{E}^{+}$, the number and the symmetrization in terms of the $2_{E}^{+}$interpolators are given for the light meson channels $2_{E}^{++}$.

\section{Baryon interpolators}

The baryon interpolators are slightly more complicated, the construction in each channel is given by:

$$
\begin{aligned}
N^{(i)} & =\epsilon_{a b c} \Gamma_{1}^{(i)} u_{a}\left(u_{b}^{T} \Gamma_{2}^{(i)} d_{c}-d_{b}^{T} \Gamma_{2}^{(i)} u_{c}\right) \\
\Sigma^{(i)} & =\epsilon_{a b c} \Gamma_{1}^{(i)} u_{a}\left(u_{b}^{T} \Gamma_{2}^{(i)} s_{c}-s_{b}^{T} \Gamma_{2}^{(i)} u_{c}\right) \\
\Xi^{(i)} & =\epsilon_{a b c} \Gamma_{1}^{(i)} s_{a}\left(s_{b}^{T} \Gamma_{2}^{(i)} u_{c}-u_{b}^{T} \Gamma_{2}^{(i)} s_{c}\right) \\
\Delta_{\mu} & =\epsilon_{a b c} u_{a}\left(u_{b}^{T} C \gamma_{\mu} u_{c}\right) \\
\Omega_{\mu} & =\epsilon_{a b c} s_{a}\left(s_{b}^{T} C \gamma_{\mu} s_{c}\right)
\end{aligned}
$$

Subsequent numbering of the interpolators with respect to gamma and smearing structure is performed in each channel, leading to the interpolator numbers given in Tables $\mathrm{X}$ and XI. Projection to definite parity is performed by the projection operator $P_{ \pm}=\left(\mathbb{1} \pm \gamma_{t}\right) / 2 . C$ is the charge conjugation operator, in the chiral representation it can be written as $C=\mathrm{i} \gamma_{2} \gamma_{4}$. The $\Delta$ and $\Omega$ interpolators are projected to spin $\frac{3}{2}$ and averaged over the three spatial vector components [44].

\begin{tabular}{ccc|c|c|c}
\hline \hline$\chi^{(i)}$ & $\Gamma_{1}^{(i)}$ & $\Gamma_{2}^{(i)}$ & Smearing & $\mathrm{N}$ & $\Sigma, \Xi$ \\
\hline$\chi^{(1)}$ & $\mathbb{1}$ & $C \gamma_{5}$ & $n(n n)$ & 1 & 1 \\
$\chi^{(1)}$ & $\mathbb{1}$ & $C \gamma_{5}$ & $n(n w)$ & 2 & 2 \\
$\chi^{(1)}$ & $\mathbb{1}$ & $C \gamma_{5}$ & $n(w n)$ & & 3 \\
$\chi^{(1)}$ & $\mathbb{1}$ & $C \gamma_{5}$ & $w(n n)$ & 3 & 4 \\
$\chi^{(1)}$ & $\mathbb{1}$ & $C \gamma_{5}$ & $n(w w)$ & 4 & 5 \\
$\chi^{(1)}$ & $\mathbb{1}$ & $C \gamma_{5}$ & $w(n w)$ & 5 & 6 \\
$\chi^{(1)}$ & $\mathbb{1}$ & $C \gamma_{5}$ & $w(w n)$ & & 7 \\
$\chi^{(1)}$ & $\mathbb{1}$ & $C \gamma_{5}$ & $w(w w)$ & 6 & 8 \\
\hline$\chi^{(2)}$ & $\gamma_{5}$ & $C$ & $n(n n)$ & 7 & 9 \\
$\chi^{(2)}$ & $\gamma_{5}$ & $C$ & $n(n w)$ & 8 & 10 \\
$\chi^{(2)}$ & $\gamma_{5}$ & $C$ & $n(w n)$ & & 11 \\
$\chi^{(2)}$ & $\gamma_{5}$ & $C$ & $w(n n)$ & 9 & 12 \\
$\chi^{(2)}$ & $\gamma_{5}$ & $C$ & $n(w w)$ & 10 & 13 \\
$\chi^{(2)}$ & $\gamma_{5}$ & $C$ & $w(n w)$ & 11 & 14 \\
$\chi^{(2)}$ & $\gamma_{5}$ & $C$ & $w(w n)$ & & 15 \\
$\chi^{(2)}$ & $\gamma_{5}$ & $C$ & $w(w w)$ & 12 & 16 \\
\hline$\chi^{(3)}$ & $\mathrm{i} \mathbb{1}$ & $C \gamma_{t} \gamma_{5}$ & $n(n n)$ & 13 & 17 \\
$\chi^{(3)}$ & $\mathrm{i} \mathbb{1}$ & $C \gamma_{t} \gamma_{5}$ & $n(n w)$ & 14 & 18 \\
$\chi^{(3)}$ & $\mathrm{i} \mathbb{1}$ & $C \gamma_{t} \gamma_{5}$ & $n(w n)$ & & 19 \\
$\chi^{(3)}$ & $\mathrm{i} \mathbb{1}$ & $C \gamma_{t} \gamma_{5}$ & $w(n n)$ & 15 & 20 \\
$\chi^{(3)}$ & $\mathrm{i} \mathbb{1}$ & $C \gamma_{t} \gamma_{5}$ & $n(w w)$ & 16 & 21 \\
$\chi^{(3)}$ & $\mathrm{i} \mathbb{1}$ & $C \gamma_{t} \gamma_{5}$ & $w(n w)$ & 17 & 22 \\
$\chi^{(3)}$ & $\mathrm{i} \mathbb{1}$ & $C \gamma_{t} \gamma_{5}$ & $w(w n)$ & & 23 \\
$\chi^{(3)}$ & $\mathrm{i} \mathbb{1}$ & $C \gamma_{t} \gamma_{5}$ & $w(w w)$ & 18 & 24 \\
\hline \hline
\end{tabular}

TABLE X: Baryon interpolators for nucleon, $\Sigma$ and $\Xi$ channels. The Dirac structures, the quark smearings and the corresponding interpolator numbers are given. $\chi^{(i)}$ labels the Dirac structure of the baryon interpolators. In the nucleon channel we prune interpolators which are very similar to others, obtaining six per Dirac structure, thus a total of 18 interpolators.

\begin{tabular}{c|c}
\hline \hline Smearing & $\Delta, \Omega$ \\
\hline$n(n n)$ & 1 \\
$n(n w)$ & 2 \\
$w(n n)$ & 3 \\
$n(w w)$ & 4 \\
$w(n w)$ & 5 \\
$w(w w)$ & 6 \\
\hline \hline
\end{tabular}

TABLE XI: Baryon interpolators for $\Delta$ and $\Omega$ channels. The quark smearings and the corresponding interpolator numbers are given. We prune interpolators which are very similar to others, obtaining six interpolators. 
[1] Particle Data Group, C. Amsler et al., Phys. Lett. B 667, 1 (2008).

[2] P. H. Ginsparg and K. G. Wilson, Phys. Rev. D 25, 2649 (1982).

[3] H. Neuberger, Phys. Lett. B 417, 141 (1998), arXiv:hep-lat/9707022.

[4] H. Neuberger, Phys. Lett. B 427, 353 (1998), arXiv:hep-lat/9801031.

[5] D. B. Kaplan, Phys. Lett. B 288, 342 (1992), arXiv:hep-lat/9206013.

[6] V. Furman and Y. Shamir, Nucl. Phys. B 439, 54 (1995), arXiv:hep-lat/9405004.

[7] P. Hasenfratz and F. Niedermayer, Nucl. Phys. B 414 785 (1994), arXiv:hep-lat/9308004.

[8] A. Hasenfratz, P. Hasenfratz and F. Niedermayer, Phys. Rev. D 72, 114508 (2005), arXiv:hep-lat/0506024.

[9] M. Lüscher, Phys. Lett. B 428, 342 (1998), arXiv:hep-lat/9802011.

[10] C. Gattringer, Phys. Rev. D 63, 114501 (2001), arXiv:hep-lat/0003005.

[11] C. Gattringer, I. Hip and C. B. Lang, Nucl. Phys. B 597, 451 (2001), arXiv:hep-lat/0007042.

[12] C. Gattringer et al., Nucl. Phys. B 677, 3 (2004), arXiv:hep-lat/0307013.

[13] C. Gattringer, P. Huber and C. B. Lang, Phys. Rev. D 72, 094510 (2005), arXiv:hep-lat/0509003.

[14] C. B. Lang, P. Majumdar and W. Ortner, Phys. Rev. D 73, 034507 (2006), arXiv:hep-lat/0512014.

[15] C. Gattringer et al., Phys. Rev. D 79, 054501 (2009), arXiv:0812.1681.

[16] C. Michael, Nucl. Phys. B 259, 58 (1985).

[17] M. Lüscher and U. Wolff, Nucl. Phys. B 339, 222 (1990).

[18] G. Engel et al., PoS LAT09, 088 (2009), arXiv:0910.2802.

[19] C. Morningstar, PoS LATTICE2008, 009 (2008), arXiv:0810.4448

[20] C. Alexandrou, C. N. Papanicolas and E. Stiliaris, PoS LATTICE2008, 099 (2008), arXiv:0810.3982.

[21] T. Burch et al., Phys. Rev. D 79, 114503 (2009), arXiv:0903.2358.

[22] T. T. Takahashi and M. Oka, PoS LAT2009, 108 (2009), arXiv:0911.2542.

[23] T. T. Takahashi and M. Oka, Phys. Rev. D 81, 034505 (2010), arXiv:0910.0686.

[24] G. T. Fleming, S. D. Cohen, H. W. Lin and V. Pereyra, Phys. Rev. D 80, 074506 (2009), arXiv:0903.2314.

[25] S. Cohen et al., Excited-Nucleon Spectroscopy with $2+1$ Fermion Flavors, 2009, arXiv:0911.3373.

[26] M. S. Mahbub et al., Phys. Rev. D 80, 054507 (2009), arXiv:0905.3616

[27] M. S. Mahbub et al., Low-lying positive-parity excited states of the nucleon, 2009, arXiv:0910.2789.

[28] M. S. Mahbub, A. O. Cais, W. Kamleh, D. B. Leinweber and A. G. Williams, Positive-parity Excited-states of the Nucleon in Lattice QCD, 2010, arXiv:1004.5455.

[29] J. J. Dudek, R. G. Edwards, M. J. Peardon, D. G. Richards and C. E. Thomas, Phys. Rev. Lett. 103, 262001 (2009), arXiv:0909.0200.

[30] J. J. Dudek, R. G. Edwards, M. J. Peardon, D. G. Richards and C. E. Thomas, Toward the excited meson spectrum of dynamical QCD, 2010, arXiv:1004.4930.

[31] J. M. Bulava et al., Phys. Rev. D 79, 034505 (2009), arXiv:0901.0027.

[32] J. Bulava et al., Nucleon, $\Delta$ and $\Omega$ excited states in $N_{f}=2+1$ lattice QCD, 2010, arXiv:1004.5072.

[33] C. Morningstar et al., The excited hadron spectrum in lattice QCD using a new method of estimating quark propagation, 2010, arXiv:1002.0818.

[34] C. Morningstar and M. Peardon, Phys. Rev. D 69, 054501 (2004), arXiv:hep-lat/0311018.

[35] S. Duane, A. D. Kennedy, B. J. Pendleton and D. Roweth, Phys. Lett. B195, 216 (1987).

[36] M. Hasenbusch, Phys. Lett. B 519, 177 (2001), arXiv:hep-lat/0107019.

[37] R. C. Brower, T. Ivanenko, A. R. Levi and K. N. Orginos, Nucl. Phys. B 484, 353 (1997), arXiv:hep-lat/9509012.

[38] S. Dürr et al., Phys. Rev. D 79, 014501 (2009), arXiv:0802.2706.

[39] C. Jung, PoS LAT2009, 002 (2010), arXiv:1001.0941.

[40] S. Aoki, Nucl. Phys. B (Proc. Suppl.) 94, 3 (2001), arXiv:hep-lat/0011074.

[41] T. Burch et al., Phys. Rev. D 70, 054502 (2004), arXiv:hep-lat/0405006.

[42] T. Burch, C. Gattringer, L. Y. Glozman, C. Hagen and C. B. Lang, Phys. Rev. D 73, 017502 (2006), arXiv:hep-lat/0511054.

[43] T. Burch et al., Phys. Rev. D 73, 094505 (2006), arXiv:hep-lat/0601026.

[44] T. Burch et al., Phys. Rev. D 74, 014504 (2006), arXiv:hep-lat/0604019.

[45] C. Gattringer, L. Y. Glozman, C. B. Lang, D. Mohler and S. Prelovsek, Phys. Rev. D 78, 034501 (2008), arXiv:0802.2020.

[46] M. Lüscher, Commun. Math. Phys. 104, 177 (1986).

[47] M. Lüscher, Commun. Math. Phys. 105, 153 (1986).

[48] M. Lüscher, Nucl. Phys. B 354, 531 (1991).

[49] M. Lüscher, Nucl. Phys. B 364, 237 (1991).

[50] B. Blossier, M. DellaMorte, G. von Hippel, T. Mendes and R. Sommer, JHEP 0904, 094 (2009), arXiv:0902.1265.

[51] L. Y. Glozman, C. B. Lang and M. Limmer, Phys. Rev. Lett. 103, 121601 (2009), arXiv:0905.0811.

[52] L. Y. Glozman, C. B. Lang and M. Limmer, Few Body Syst. 47, 91 (2010), arXiv:0909.2939.

[53] S. Güsken et al., Phys. Lett. B 227, 266 (1989).

[54] C. Best et al., Phys. Rev. D 56, 2743 (1997), arXiv:hep-lat/9703014.

[55] C. Gattringer et al., PoS LATTICE2008, 093 (2008), arXiv:0809.4514.

[56] A. Hasenfratz, R. Hoffmann and F. Knechtli, Nucl. Phys. B (Proc. Suppl.) 106, 418 (2002), arXiv:hep-lat/0110168.

[57] A. Hasenfratz and F. Knechtli, Phys. Rev. D 64, 034504 (2001), arXiv:hep-lat/0103029.

[58] A. Hasenfratz, R. Hoffmann and S. Schaefer, JHEP 05, 029 (2007), arXiv:hep-lat/0702028.

[59] M. Peardon et al., Hadron Spectrum Collaboration, Phys. Rev. D 80, 054506 (2009), arXiv:0905.2160.

[60] P. Lacock, C. Michael, P. Boyle and P. Rowland, UKQCD Collaboration, Phys. Rev. D 54, 6997 (1996), 
arXiv:hep-lat/9605025.

[61] X. Liao and T. Manke, Excited charmonium spectrum from anisotropic lattices, 2002, arXiv:hep-lat/0210030.

[62] S. Basak et al., Phys. Rev. D 72, 094506 (2005), arXiv:hep-lat/0506029.

[63] J. J. Dudek, R. G. Edwards, N. Mathur and D. G. Richards, Phys. Rev. 77, 034501 (2008), arXiv:0707.4162.

[64] R. G. Petry, D. Harnett, R. Lewis and R. M. Woloshyn, Phys. Rev. D 78, 074502 (2008), arXiv:0803.4141.

[65] S. Prelovsek, C. Dawson, T. Izubuchi, K. Orginos and A. Soni, Phys. Rev. D 70, 094503 (2004), arXiv:hep-lat/0407037.

[66] N. Mathur et al., Phys. Rev. D 76, 114505 (2007), arXiv:hep-ph/0607110.

[67] S. Prelovsek and D. Mohler, Phys. Rev. D 79, 014503 (2009), arXiv:0810.1759.

[68] C. R. Gattringer and C. B. Lang, Nucl. Phys. B 391, 463 (1993).

[69] D. C. Moore and G. T. Fleming, Phys. Rev. D 74, 054504 (2006), arXiv:hep-lat/0607004.

[70] J. Bulava, K. J. Juge, C. J. Morningstar, M. J. Peardon and C. H. Wong, Two-particle Correlation Functions with Distilled Propagators, 2009, arXiv:0911.2044.

[71] G. Bali and C. Ehmann, Mixing of S-Wave Charmonia

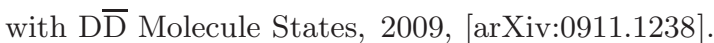

[72] S. Prelovsek et al., PoS LAT2009, 103 (2009), arXiv:0910.2749.

[73] S. Prelovsek et al., Lattice study of light scalar tetraquarks with $\mathrm{I}=0,2,1 / 2,3 / 2$ : are sigma and kappa tetraquarks?, 2010, arXiv:1005.0948.

[74] S. Weinberg, Physica A 96, 327 (1979).

[75] J. Gasser and H. Leutwyler, Nucl. Phys. B 250, 465 (1985).

[76] A. K. De, A. Harindranath and J. Maiti, On Scale Determination in Lattice QCD with Dynamical Quarks, 2008, arXiv:0803.1281.

[77] S. Dürr et al., $\quad$ Science 322, 1224 (2008), arXiv:0906.3599.

[78] K. Jansen, C. Michael and C. Urbach, Eur. Phys. J. C 58, 261 (2008), arXiv:0804.3871.

[79] K. Jansen, C. McNeile, C. Michael and C. Urbach, Phys. Rev. 80, 054510 (2009), arXiv:0906.4720.

[80] A. Walker-Loud et al., Phys. Rev. D 79, 054502 (2009), arXiv:0806.4549.

[81] A. Walker-Loud, PoS LATTICE2008, 005 (2008), arXiv:0810.0663.

[82] T. T. Takahashi and T. Kunihiro, Phys. Rev. D 78, 011503(R) (2008), arXiv:0801.4707. 